International Electronic Journal of Algebra

Volume 31 (2022) 13-37

DOI: $10.24330 /$ ieja. 1058385

\title{
ON LATTICES ASSOCIATED TO RINGS WITH RESPECT TO A
} PRERADICAL

\author{
Erwin Cerda-León and Hugo Rincón-Mejía
}

Received: 1 August 2020; Revised: 14 May 2021; Accepted: 21 May 2021

Communicated by Christian Lomp

\begin{abstract}
We introduce some new lattices of classes of modules with respect to appropriate preradicals. We introduce some concepts associated with these lattices, such as the $\sigma$-semiartinian rings, the $\sigma$-retractable modules, the $\sigma$ $V$-rings, the $\sigma$-max rings. We continue to study $\sigma$-torsion theories, $\sigma$-open classes, $\sigma$-stable classes. We prove some theorems that extend some known results. Our results fall into well known situations when the preradical $\sigma$ is chosen as the identity preradical.
\end{abstract}

Mathematics Subject Classification (2020): 16W30

Keywords: Preradical, lattice of module class, hereditary torsion class, boolean lattice, V-ring, max-ring

\section{Introduction}

Lattices and big lattices of module classes has been studied to obtain information about the underlying ring $R$ and about its associated module category. For example, the big lattice of preradicals and some associated lattices of special kinds of preradicals have provided a wealth of information about the rings and their module categories.

Similar considerations can be made about module classes lattices defined by closure properties. Some examples of these lattices are: the lattice of the natural classes, the lattice of the hereditary torsion classes, the lattice of Serre classes, that of the Wisbauer classes and some others.

In [6], the big lattices of module classes induced by a preradical $\sigma$ over $R$-Mod were introduced, for example, the lattices of $\sigma$-hereditary classes, of $\sigma$-cohereditary classes, of $\sigma$-natural classes, and of $\sigma$-conatural classes. Note that the $\sigma$-open classes lattice and the $\sigma$-torsion theories lattice were also introduced in the same paper.

Our objective in this work is to introduce some new lattices of module classes with respect to a preradical $\sigma$, to use these lattices to set properties for rings and for

This research was supported by a grant from the PostDoctoral Scholarship Program at the National Autonomous University of Mexico. 
their module categories. We introduce the $\sigma$-semiartinian rings, the $\sigma$-retractable modules, $\sigma$ - $V$-rings, and $\sigma$-max rings. We extend some well known results in the literature.

\section{Preliminaries}

2.1. Preradicals and classes of modules. In this section, we present basic results about preradicals on $R$-Mod and about classes of modules. For more information about preradicals, see [5], [11] and [14]. We refer to [1], [4], and [7], for basic results about hereditary, cohereditary, natural, conatural and open classes.

A preradical on $R$-mod is an assignment $\sigma: R$-Mod $\rightarrow R$-Mod such that for each $M \in R$-Mod, $\sigma(M) \leq M$ and for each $R$-morphism $f: M \rightarrow N, f(\sigma(M)) \leq \sigma(N)$. $\sigma$ is a subfunctor of the identity functor on $R$-Mod. $R$-pr denotes the collection of all preradicals on $R$-Mod.

In $R$-pr, we have two binary operations, one of them called the product, denoted with "." and the other called the coproduct denoted with ":" given by:

$$
\begin{aligned}
& (\sigma \cdot \tau)(M)=\sigma(\tau(M)) \\
& (\sigma: \tau)(M) \text { is defined by }(\sigma: \tau)(M) / \sigma(M)=\tau(M / \sigma(M))
\end{aligned}
$$

We will write $\sigma \tau$ instead of $\sigma \cdot \tau$. A preradical $\sigma$ is called idempotent if $\sigma \sigma=\sigma$, and $\sigma$ is called a radical if $(\sigma: \sigma)=\sigma$.

Let us recall that $\sigma$ is a radical if and only if $\sigma(M / \sigma(M))=0$, for each $M \in R$ Mod. $\sigma$ is t-radical if and only if $\sigma(M)=\sigma(R) M$. $t$-radicals are precisely the preradicals preserving epimorphisms. $t$-radicals are also called cohereditary radicals.

A preradical $\sigma$ is a left exact preradical if it is a left exact functor. This is equivalent to the condition that for each submodule $N$ of a module $M$ we have that $\sigma(N)=N \cap \sigma(M)$. That $\sigma$ is a left exact preradical it is also equivalent to that $\sigma$ is an idempotent preradical and $\mathbb{T}_{\sigma}$ is a hereditary class.

We will denote $R$-id, $R$-rad, $R$-lep, $R$-radid the collections of idempotent preradicals, of radicals, of left exact preradicals, and of idempotent radicals, respectively.

Each preradical $\sigma$ has associated the class $\mathbb{T}_{\sigma}=\{M \in R$-Mod $\mid \sigma(M)=M\}$. This class is closed under quotients and direct sums and it is called the $\sigma$-pretorsion class.

Let us recall that a class of $R$-modules is a pretorsion free class if it is closed under taking submodules and direct products. Each $\sigma \in R$-pr has associated the pretorsion free class $\mathbb{F}_{\sigma}=\{N \in R$-Mod $\mid \sigma(N)=0\}$.

We say that a module $M$ splits in a preradical $\sigma$ if $M=\sigma(M) \oplus M^{\prime}$ for some $M^{\prime} \leq$ $M$. Notice that in this case, $\sigma\left(M^{\prime}\right)=0, \sigma(\sigma(M))=\sigma(M)$ and $\sigma(M / \sigma(M))=0$. 
$\sigma \in R$-pr is stable (costable) if for each injective (projective) module $Q, Q$ splits in $\sigma$. This implies that $\sigma(Q)$ is an injective (projective) module. We say that $\sigma$ centrally splits if for each $R$-module $M$ we have that $M=\sigma(R) M \oplus M^{\prime}$, with $M^{\prime}=\{m \in M \mid \sigma(R) m=0\}$, for further information, see [5], Chapter I.

We say that a two sided ideal $I$ of a ring $R$ is pure if $I J=I \cap J$ for every ideal $J$ of $R$. For a two sided ideal $I$ we have that $I$ is a pure ideal $\Leftrightarrow$ for each $M \in R$-Mod and $N \leq M, I N=N \cap I M \Leftrightarrow R / I$ is a flat module (see [14] Chap. I, §11) $\Leftrightarrow$ for all $a \in I, a \in I a$. Notice that if $I$ is a pure ideal, then the preradical $I \cdot-$ is exact.

Remark 2.1. ([14], page 157) Take $\sigma \in R$-pr. The following conditions are equivalent:

(1) $\sigma$ is a $t$-radical.

(2) $\sigma$ preserves epimorphisms.

(3) $\sigma$ is a radical and $\mathbb{F}_{\sigma}$ is closed under quotient modules.

If $\sigma$ is an exact preradical, then $\sigma$ is a t-radical and $\sigma(R)$ is a pure ideal because for each $M \in R$-Mod and $N \leq M$ we have that $\sigma(R) N=\sigma(N)=N \cap \sigma(M)=$ $N \cap \sigma(R) M$.

If $I \leq R$ is a pure ideal, then $I$ defines an exact preradical $\sigma$ by $\sigma(M)=I M$.

2.2. Classes of modules. A lattice $L$ is bounded if it has a smallest element (usually denoted by $\mathbf{0}$ ) and a largest element (usually denoted by $\mathbf{1}$ ). In a lattice $L$ with $\mathbf{0}$, an element $a^{*}$ is a pseudocomplement of $a \in L$, if $a \wedge a^{*}=0$ and $a^{*}$ is maximal in $L$ with respect to this property. We say that $a^{*}$ is a strong pseudocomplement of $a$ if it is the largest element in $L$ with respect to $a \wedge a^{*}=0$.

We will denote $\operatorname{Skel}(L)=\left\{a^{*} \mid a \in L\right\}$ and we will call it the skeleton of $L$. In a bounded lattice $L$, we will say that $a^{*} \in L$ is a complement of $a \in L$ if $a^{*} \wedge a=0$ and $a^{*} \vee a=1$.

If $L$ is a proper class instead of a set, we will say that $L$ is a big lattice.

A class of left $R$-modules is called an abstract class if it is closed under taking isomorphic copies of its members. We consider some closure properties of a class of modules, like being closed under submodules, quotients, extensions, direct sums, injective hulls, products or projective covers, we will use the symbols $\leq, \rightarrow$, ext $\oplus, E, \prod, P$ respectively, to abbreviate. If $A$ denotes a set of these closure properties, we denote $\mathscr{L}_{A}$ the proper class of classes of modules closed under each closure property in $A$. So, $\mathscr{L}_{\{\leq\}}$denotes the proper class of hereditary classes in R-mod, $\mathscr{L}_{\{\leq, \oplus, E\}}$ denotes the class of natural classes, and so on. 
We should notice that $\mathscr{L}_{A}$ becomes a complete big with inclusion of classes as the order and with infima given by intersections. We will denote $\xi_{A}(\mathscr{C})$ the least class in $\mathscr{L}_{A}$ containing $\mathscr{C}$ and by $\chi_{A}(\mathscr{C})$ the largest class in $\mathscr{L}_{A}$ contained in $\mathscr{C}$. Thus $\xi_{\{\leq\}}(\mathscr{C})$ denotes the hereditary closure of $\mathscr{C}$, and $\xi_{\{\rightarrow\}}(\mathscr{C})$ denotes the homomorphic image closure of $\mathscr{C} . \xi_{\{\leq\}}(\mathscr{C})$ will be denoted also $S(\mathscr{C})$ and $\xi_{\{\rightarrow\}}(\mathscr{C})$ will be denoted also $H(\mathscr{C})$.

The big lattice of torsion theories is denoted by $R$-TORS (see [14], Chapter VI), and the lattice of hereditary torsion theories is denoted by $R$-tors (see [9]). Often it will be convenient to identify each torsion theory with its torsion class, that is, $R$-TORS $=\mathscr{L}_{\{\rightarrow, \oplus, e x t\}}$ and $R$-tors $=\mathscr{L}_{\{\leq, \rightarrow, \oplus, e x t\}}$. We denote $R$-jtors $=\{\mathscr{C} \in$ $R$-tors $\left.\mid \mathscr{C} \in \mathscr{L}_{\{\Pi\}}\right\}$.

For a module class $\mathfrak{a}$, we denote $\xi(\mathfrak{a})$ the least hereditary torsion theory containing $\mathfrak{a}$, and by $\chi(\mathfrak{a})$ the largest hereditary torsion theory such that each one of its modules has no nonzero submodules in $\mathfrak{a}$.

Remark 2.2. $\wp(R$-Mod $):=\{\mathscr{C} \mid \mathscr{C} \subseteq R$-Mod $\}$. Each $\sigma \in R$-pr define two assignments:

(1) $\sigma^{*}: \wp(R$-Mod $) \rightarrow \wp(R$-Mod $)$, where $\sigma^{*}(\mathscr{C})=\{\sigma(M) \mid M \in \mathscr{C}\}$.

$(2) \overleftarrow{\sigma}: \wp(R$-Mod $) \rightarrow \wp(R$-Mod $)$, where $\overleftarrow{\sigma}(\mathscr{C})=\{M \in R$-Mod $\mid \sigma(M) \in \mathscr{C}\}$

Notice that $\sigma^{*}\left(\overleftarrow{\sigma}\left(\sigma^{*}(\mathscr{C})\right)\right)=\sigma^{*}(\mathscr{C})$ and $\overleftarrow{\sigma}\left(\sigma^{*}(\overleftarrow{\sigma}(\mathscr{C}))\right)=\overleftarrow{\sigma}(\mathscr{C})$ for each $\mathscr{C} \subseteq$ $R$-Mod.

\section{3. $\sigma$-(R-tors $)$ and $\sigma$-(R-TORS $)$}

Let us take a preradical $\sigma$. We will say that a class $\mathscr{C} \subseteq R$-Mod is $\sigma$-hereditary ( $\sigma$-cohereditary) if it has the following two conditions: $\mathbb{F}_{\sigma} \subseteq \mathscr{C}$, and for each $M \in \mathscr{C}$ and $N \leq M(M \rightarrow N)$ it happens that $\sigma(N) \in \mathscr{C}$. We denote $\mathscr{L}_{\left\{\leq_{\sigma}\right\}}\left(\mathscr{L}_{\{\rightarrow \sigma\}}\right)$ the collection of all hereditary $\sigma$-hereditary $\left(\sigma\right.$-cohereditary) classes. $\mathscr{L}_{\left\{\leq_{\sigma}\right\}}$ is a bounded pseudocomplemented big lattice, whose least member is $\mathbb{F}_{\sigma}$ and whose largest member is $R$-Mod, where infima is given by class intersections. If $\sigma$ is an idempotent preradical then the pseudocomplements are strong and $\operatorname{Skel}\left(\mathscr{L}_{\left\{\leq_{\sigma}\right\}}\right)$ is a boolean lattice. If $\sigma$ is an idempotent cohereditary preradical, then $\mathscr{L}_{\{\rightarrow \sigma\}}$ is a strongly pseudocomplemented big lattice. The big lattice of $\sigma$-open classes is denoted by $\mathscr{L}_{\left\{\leq_{\sigma}, \rightarrow_{\sigma}\right\}}$. If $\sigma$ is an exact preradical, then $\operatorname{Skel}\left(\mathscr{L}_{\left\{\leq_{\sigma}, \rightarrow_{\sigma}\right\}}\right)=\{\overleftarrow{\sigma}$ $\left.(\mathscr{C}) \mid \mathscr{C} \in \operatorname{Skel}\left(\mathscr{L}_{\{\leq, \rightarrow\}}\right)\right\}$ (see $\left.[6]\right)$.

Definition 3.1. Let $\sigma$ be a preradical.

(1) $R$ - $(\sigma$-TORS $):=\mathscr{L}_{\{\rightarrow \sigma, \oplus, e x t\}}$. 
(2) $R$-( $\sigma$-tors $):=\mathscr{L}_{\left\{\leq_{\sigma}, \rightarrow_{\sigma}, \oplus, e x t\right\}}$.

(3) $\mathscr{L}_{\{\sigma P\}}=\{\mathscr{C} \subseteq R$-Mod $\mid \forall M \in \mathscr{C}$, and for each projective cover $g$ : $P(M) \rightarrow M, \sigma(P(M)) \in \mathscr{C}\}$.

(4) $\mathscr{L}_{\{\sigma E\}}=\{\mathscr{C} \subseteq R$-Mod $\mid \forall M \in \mathscr{C}, \sigma(E(M)) \in \mathscr{C}\}$.

Remark 3.2. If $\mathscr{C} \subseteq R$-Mod, then

$$
\begin{aligned}
& \xi_{\{\leq\}}(\mathscr{C})=\{N \in R \text {-Mod } \mid \exists N \longmapsto M, M \in \mathscr{C}\}, \\
& \xi_{\{\rightarrow\}}(\mathscr{C})=\{L \in R \text {-Mod } \mid \exists M \rightarrow L, M \in \mathscr{C}\} .
\end{aligned}
$$

Lemma 3.3. Let $\sigma$ be a radical and $\mathscr{C} \in \mathscr{L}_{\{\text {ext }\}}$ with $\mathscr{C} \supseteqq \mathbb{F}_{\sigma}$, then $\overleftarrow{\sigma}(\mathscr{C}) \subseteq \mathscr{C}$

Proof. Take $\sigma$ and $\mathscr{C}$ as in the statement. If $A \in \overleftarrow{\sigma}(\mathscr{C})$ then we have the exact sequence

$$
0 \rightarrow \sigma(A) \rightarrow A \rightarrow A / \sigma(A) \rightarrow 0
$$

with $\sigma(A) \in \mathscr{C}$ and with $A / \sigma(A) \in \mathbb{F}_{\sigma} \subseteq \mathscr{C}$. Thus $A \in \mathscr{C}$.

Lemma 3.4. If $\mathscr{C} \in \mathscr{L}_{\{\leq\}}$, then $\mathscr{C} \subseteq \overleftarrow{\sigma}(\mathscr{C})$

Proof. Let $\mathscr{C} \in \mathscr{L}_{\{\leq\}}$and $M \in \mathscr{C}$, if $N \leq M$ then $N \in \mathscr{C}$, thus $\sigma(M) \in \mathscr{C}$ so that $M \in \overleftarrow{\sigma}(\mathscr{C})$

From the two previous lemmas it follows the following remark.

Remark 3.5. If $\mathscr{C} \in \mathscr{L}_{\{\leq, e x t\}}$ and $\sigma$ is a radical with $\mathbb{F}_{\sigma} \subseteq \mathscr{C}$, then $\mathscr{C}=\overleftarrow{\sigma}(\mathscr{C})$

Theorem 3.6. Let $\sigma \in R$-pr:

(1) $\mathscr{C} \in \mathscr{L}_{\left\{\leq_{\sigma}\right\}} \Leftrightarrow \xi_{\leq}(\mathscr{C}) \subseteq \overleftarrow{\sigma}(\mathscr{C})$

(2) $\mathscr{C} \in \mathscr{L}_{\left\{\rightarrow_{\sigma}\right\}} \Leftrightarrow \xi_{\rightarrow}(\mathscr{C}) \subseteq \overleftarrow{\sigma}(\mathscr{C})$

Proof. (1) $(\Rightarrow)$ : Suppose that $\mathscr{C} \in \mathscr{L}_{\left\{\leq_{\sigma}\right\}}$ and $N \in \xi_{\leq}(\mathscr{C})$. Then there is a monomorphism $N \longmapsto M$ with $M \in \mathscr{C}$. Thus $\sigma(N) \in \mathscr{C}$, and $N \in \overleftarrow{\sigma}(\mathscr{C})$.

$(\Leftarrow)$ : Suppose that $N \leq M$ with $M \in \mathscr{C}$, then $N \in \xi_{\leq}(\mathscr{C}) \subseteq \overleftarrow{\sigma}(\mathscr{C})$. Hence $\sigma(N) \in \mathscr{C}$. As it is clear that $\mathbb{F}_{\sigma} \subseteq \mathscr{C}$, we have that $\mathscr{C} \in \mathscr{L}_{\left\{\leq_{\sigma}\right\}}$.

(2) ( $\Rightarrow$ ): Let us take $\mathscr{C} \in \mathscr{L}_{\{\rightarrow \sigma\}}$ and $L \in \xi_{\rightarrow}(\mathscr{C})$, there is an epimorphism $M \rightarrow L$ with $M \in \mathscr{C}$. Then $\sigma(L) \in \mathscr{C}$, hence $L \in \overleftarrow{\sigma}(\mathscr{C})$.

$(\Leftarrow)$ : Let us assume $\xi_{\rightarrow}(\mathscr{C}) \subseteq \overleftarrow{\sigma}(\mathscr{C})$ and take an epimorphism $M \rightarrow L$ with $M \in \mathscr{C}$. Then $L \in \overleftarrow{\sigma}(\mathscr{C})$, thus $\sigma(L) \in \mathscr{C}$. Also, it is clear that $\mathbb{F}_{\sigma} \subseteq \mathscr{C}$, thus we have that $\mathscr{C} \in \mathscr{L}_{\{\rightarrow \sigma\}}$.

Theorem 3.7. If $\sigma$ is a radical then $\mathscr{L}_{\left\{\leq_{\sigma}, e x t\right\}}=\left\{\mathscr{C} \in \mathscr{L}_{\{\leq, e x t\}} \mid \mathscr{C} \supseteq \mathbb{F}_{\sigma}\right\}$. 
Proof. (つ): If $\mathscr{C} \in \mathscr{L}_{\{\leq, e x t\}}$ and $\mathscr{C} \supseteq \mathbb{F}_{\sigma}$, then $\mathscr{C}=\overleftarrow{\sigma}(\mathscr{C})$, by Remark 3.5. Thus $\xi_{\{\leq\}}(\mathscr{C})=\mathscr{C}=\overleftarrow{\sigma}(\mathscr{C})$. Then $\mathscr{C} \in \mathscr{L}_{\left\{\leq_{\sigma}\right\}}$, by Theorem 3.6.

$(\subseteq)$ : If $\mathscr{C} \in \mathscr{L}_{\left\{\leq_{\sigma}, e x t\right\}}$, then $\mathbb{F}_{\sigma} \subseteq \mathscr{C}$ and $\mathscr{C} \subseteq \xi_{\{\leq\}}(\mathscr{C}) \subseteq \overleftarrow{\sigma}(\mathscr{C}) \subseteq \mathscr{C}$, by Theorem 3.6 and Lemma 3.3. Thus $\mathscr{C}=\xi_{\{\leq\}}(\mathscr{C})$ and $\mathscr{C} \in \mathscr{L}_{\{\leq, e x t\}}$.

Next corollary follows immediately.

Corollary 3.8. If $\sigma$ is a radical then $\mathscr{L}_{\left\{\leq_{\sigma}, \text { ext }, \oplus\right\}}=\left\{\mathscr{C} \in \mathscr{L}_{\{\leq, e x t, \oplus\}} \mid \mathscr{C} \supseteq \mathbb{F}_{\sigma}\right\}$.

Theorem 3.9. If $\sigma$ is a radical then

$$
\mathscr{L}_{\left\{\leq_{\sigma}, e x t, \rightarrow_{\sigma}\right\}}=\left\{\mathscr{C} \in \mathscr{L}_{\{\leq, e x t, \rightarrow\}} \mid \mathscr{C} \supseteq \mathbb{F}_{\sigma}\right\} .
$$

Proof. (つ): It suffices to show that a class $\mathscr{C}$ belonging to the left class is cohereditary. If $M \rightarrow N$ is an epimorphism with $M \in \mathscr{C}$, then $\sigma(N) \in \mathscr{C}$. From the exact sequence

$$
0 \rightarrow \sigma(N) \rightarrow N \rightarrow N / \sigma(N) \rightarrow 0,
$$

where $N / \sigma(N) \in \mathbb{F}_{\sigma} \subseteq \mathscr{C}$, we see that $N \in \mathscr{C}$.

$(\subseteq)$ : Suppose $\mathscr{C}$ is a module class with the following properties: hereditary, cohereditary, closed under extensions and containing $\mathbb{F}_{\sigma}$. We want to prove that $\mathscr{C}$ is $\sigma$-hereditary and $\sigma$-cohereditary. First, we show that it is $\sigma$-hereditary. If $N \leq M$ with $M \in \mathscr{C}$, then $\sigma(N) \leq N \leq M$, thus $\sigma(N) \in C$. Now we are going to see that $\mathscr{C}$ is also $\sigma$-cohereditary. If $M \rightarrow N$ is an epimorphism with $M \in \mathscr{C}$, then $N$ belongs to $\mathscr{C}$ and so does $\sigma(N)$.

Corollary 3.10. If $\sigma$ is a radical, then

$$
R \text { - }(\sigma \text {-tors })=\left\{\mathscr{C} \in R \text {-tors } \mid \mathscr{C} \supseteq \mathbb{F}_{\sigma}\right\} .
$$

Proof. As $R$-( $\sigma$-tors $):=\mathscr{L}_{\left\{\leq_{\sigma}, \rightarrow \sigma, \oplus, e x t\right\}}$, we have that $R$-( $\sigma$-tors $)$ consists of the module classes belonging to

$$
\left\{\mathscr{C} \in \mathscr{L}_{\{\leq, e x t, \rightarrow\}} \mid \mathscr{C} \supseteq \mathbb{F}_{\sigma}\right\}
$$

which are closed under direct sums. Thus the result follows immediately from the preceding theorem.

Example 3.11. If $R=S \times T$ with $S$ and $T$ two rings. Define $\sigma \in R$-pr by $\sigma(M)=e M$, where $e=(1,0)$. If $(\mathbb{T}, \mathbb{F})$ is a torsion theory in $S$-Mod, then $(\mathbb{T} \times$ $T$-Mod, $\mathbb{F} \times T$-Mod) is a $\sigma$-torsion theory in $R$-Mod.

Lemma 3.12. Let $\sigma$ be a preradical. Then $\mathscr{L}_{\leq_{\sigma}} \supseteq\left\{\overleftarrow{\sigma}(\mathscr{C}) \mid \mathscr{C} \in \mathscr{L}_{\leq}\right\}$ 
Proof. Let $\mathscr{C}$ be a hereditary module class, we want to show that $\overleftarrow{\sigma}(\mathscr{C})$ is a $\sigma$-hereditary class. If $N \rightarrow M$ is a monomorphism with $M \in \overleftarrow{\sigma}(\mathscr{C})$ then $\sigma(N)$ embeds in $\sigma(M)$. As $\sigma(M) \in \mathscr{C}$. then $\sigma(N)$ and $\sigma(\sigma(N))$ belong to $\mathscr{C}$. Therefore $\sigma(N) \in \overleftarrow{\sigma}(\mathscr{C})$

Theorem 3.13. Let $\sigma$ be an exact preradical. Then

$$
R-(\sigma \text {-TORS })=\{\overleftarrow{\sigma}(\mathscr{C}) \mid \mathscr{C} \in R \text {-TORS }\}
$$

Proof. (): Let us assume $\mathscr{C} \in R$-TORS, we are going to show that $\overleftarrow{\sigma}(\mathscr{C}) \in$ $R$ - $(\sigma$-TORS $)$.

If $M \rightarrow N$ is an epimorphism with $M \in \overleftarrow{\sigma}(\mathscr{C})$, let us see that $\sigma(N) \in \overleftarrow{\sigma}(\mathscr{C})$

As $M \in \overleftarrow{\sigma}(\mathscr{C})$, then $\sigma(M) \in \mathscr{C}$. As $f$ is an epimorphism, $\sigma$ is a radical and $\mathscr{C}$ is closed under quotients, then $\sigma(M) \in \mathscr{C}$. As $\sigma$ is idempotent, then $\sigma(N)=$ $\sigma(\sigma(N)) \in \mathscr{C}$. Hence $\sigma(N) \in \overleftarrow{\sigma}(\mathscr{C})$

To show that $\overleftarrow{\sigma}(\mathscr{C})$ is closed under extensions, let us assume that

$$
0 \rightarrow A \rightarrow B \rightarrow C \rightarrow 0
$$

is an exact sequence with $A, B \in \overleftarrow{\sigma}(\mathscr{C})$. Then

$$
0 \rightarrow \sigma(A) \rightarrow \sigma(B) \rightarrow \sigma(C) \rightarrow 0
$$

is also exact with $\sigma(A), \sigma(C) \in \mathscr{C}$. As $\mathscr{C} \in R$-TORS, then $\sigma(B) \in \mathscr{C}$. I.e. $B \in \overleftarrow{\sigma}$ $(\mathscr{C})$.

If $\left\{M_{i}\right\}_{I}$ is a family in $\overleftarrow{\sigma}(\mathscr{C})$ then $\sigma\left(\bigoplus M_{i}\right)=\bigoplus \sigma\left(M_{i}\right) \in \mathscr{C}$, thus $\bigoplus M_{i} \in \overleftarrow{\sigma}$ $(\mathscr{C})$.

$(\subseteq):$ If $\mathscr{C} \in R$-( $(\sigma$-TORS $)$, we are going to show that $\mathscr{C}=\overleftarrow{\sigma}\left(\sigma^{*}(\mathscr{C})\right)$, and that $\sigma^{*}(\mathscr{C}) \in R$-TORS.

Clearly, $\mathscr{C} \subseteq \overleftarrow{\sigma}\left(\sigma^{*}(\mathscr{C})\right)$. If $\sigma(M)=\sigma(C)$ with $C \in \mathscr{C}$, then $\sigma(C) \in \mathscr{C}$. As $\sigma(M), M / \sigma(M)$ both belong to $\mathscr{C}$ because $\sigma$ is a radical and $\mathbb{F}_{\sigma} \subseteq \mathscr{C}$, from $0 \rightarrow \sigma(M) \rightarrow M \rightarrow M / \sigma(M) \rightarrow 0$ we obtain that $M \in \mathscr{C}$.

It remains to show that $\sigma^{*}(\mathscr{C}) \in R$-( $\sigma$-TORS).

If $M \in \mathscr{C}$ and $\sigma(M) \rightarrow N$ is an epimorphism, from the diagram

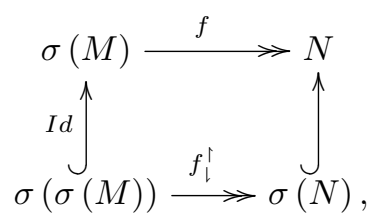

we get that $\sigma(N)=N \in \mathscr{C}$. Hence $N=\sigma(N) \in \sigma^{*}(\mathscr{C})$. 
If $0 \rightarrow \sigma(A) \rightarrow \sigma(B) \rightarrow \sigma(C) \rightarrow 0$ is an exact sequence with $A, C \in \mathscr{C}$, then we have also $\sigma(A), \sigma(C) \in \mathscr{C}$ and we obtain that $\sigma(B) \in \mathscr{C}$. Then $\sigma(B)=\sigma(\sigma(B)) \in$ $\sigma^{*}(\mathscr{C})$.

If $\left\{\sigma\left(M_{i}\right)\right\}_{I}$ is a family with $M_{i} \in \mathscr{C} \in R$-( $\sigma$-TORS $), \forall i \in I$, then also $\sigma\left(M_{i}\right) \in$ $\mathscr{C}, \forall i \in I$. Then $\sigma\left(\oplus M_{i}\right)=\oplus \sigma\left(M_{i}\right) \in \mathscr{C}$, with $\oplus M_{i} \in \mathscr{C}$. Hence $\oplus \sigma\left(M_{i}\right) \in$ $\sigma^{*}(\mathscr{C})$.

Recall that for each $\mathscr{C} \in R$-Mod, if we define

$$
\begin{gathered}
l(\mathscr{C})=\left\{M \in R \text {-Mod } \mid \operatorname{Hom}_{R}(M, E(N))=0, \forall N \in \mathscr{C}\right\} \text { and } \\
r(\mathscr{C})=\left\{N \in R \text {-Mod } \mid \operatorname{Hom}_{R}(M, E(N))=0, \forall M \in \mathscr{C}\right\},
\end{gathered}
$$

then $l(\mathscr{C})$ is a hereditary torsion class and $r(\mathscr{C})$ is its corresponding hereditary torsion free class (see [14] Chap. VI).

Recall that $R$-Simp denotes a set of representatives of isomorphism classes of simple modules.

An $R$-module $M$ is semiartinian if and only if $M$ is of $\xi(R$-Simp)-torsion if and only if each one of its nonzero homomorphic images has a nonzero socle. $R$ is a left semiartinian ring if it is semiartinian as a left $R$-module (See [9], Chap. 36).

Theorem 3.14. ([9], Chap. 36, Prop. 36.4) The following conditions are equivalent for a ring $R$.

(1) $R$ is a left semiartinian ring.

(2) Each hereditary torsion theory in R-Mod is generated by a family of simple modules.

(3) $R$-tors is a boolean lattice.

Remark 3.15. If $R$-tors $=\operatorname{Skel}\left(\mathscr{L}_{\{\leq, \rightarrow\}}\right)$ (which happens if and only if $R$ is left semiartinian), then for each $\mathbb{T} \in R$-tors there exists $\mathfrak{a} \subseteq R$-Simp such that $\mathbb{T}=$ $\mathbb{T}_{\chi(\mathfrak{a})}$. Thus, if $R$ - $(\sigma$-tors $)=\operatorname{Skel}\left(\mathscr{L}_{\left\{\leq_{\sigma}, \rightarrow_{\sigma}\right\}}\right)$, then $R$ - $(\sigma$-tors $)=\left\{\overleftarrow{\sigma}\left(\mathbb{T}_{\chi(\mathfrak{a})}\right) \mid \mathfrak{a} \subseteq\right.$ $R$-Simp\}.

Furthermore, for each $\mathfrak{a} \subseteq R$-Simp we have that $\mathbb{T}_{\xi(\mathfrak{a})}=\mathbb{T}_{\chi(R \text {-Simp } \backslash \mathfrak{a})}$.

Remark 3.16. For each centrally splitting preradical $\sigma$, we have that $\mathbb{T}_{\sigma}=$ $\sigma(R)$-Mod.

For each $M \in \mathbb{T}_{\sigma}$ there exists an epimorphism $R^{(X)} \rightarrow M$ for some set $X$. As $\sigma$ is centrally splitting we have that $\sigma(R)^{(X)} \rightarrow \sigma(M)$ is an epimorphism, thus $M=\sigma(M) \in \sigma(R)$-Mod. So $\mathbb{T}_{\sigma} \subseteq \sigma(R)$-Mod.

For the other inclusion, notice that for each $M \in \sigma(R)$-Mod there exists an epimorphism $g: \sigma(R)^{(X)} \rightarrow M$ for some set $X$, thus $M=g\left(\sigma\left(R^{(X)}\right)\right) \leq \sigma(M)$. Hence $M \in \mathbb{T}_{\sigma}$. Therefore $\sigma(R)$-Mod $\subseteq \mathbb{T}_{\sigma}$. 
Furthermore, for each $\mathscr{C} \in R$-( $\sigma$-tors $)$ we have that $\sigma^{*}(\mathscr{C}) \subseteq \sigma(R)$-Mod and $\sigma^{*}(\mathscr{C}) \in \sigma(R)$-tors.

Proposition 3.17. Let $\sigma$ be a centrally splitting preradical. The assignment $\sigma^{*}$ : $R$ - $(\sigma$-tors $) \rightarrow R$-tors satisfies $\sigma^{*}(\mathscr{C} \cap \mathscr{D})=\sigma^{*}(\mathscr{C}) \cap \sigma^{*}(\mathscr{D})$ for each $\mathscr{C}, \mathscr{D} \in$ $R$-( $\sigma$-tors $)$. Then $\sigma^{*}: R$-( $\sigma$-tors $) \rightarrow \sigma(R)$-tors is a $\wedge$-isomorphism.

Proof. Take $\mathscr{C}, \mathscr{D} \in R$-( $\sigma$-tors $)$. We have that $\sigma^{*}(\mathscr{C} \cap \mathscr{D}) \subseteq \sigma^{*}(\mathscr{C}) \cap \sigma^{*}(\mathscr{D})$. If $M \in \sigma^{*}(\mathscr{C}) \cap \sigma^{*}(\mathscr{D})$, then $M \in \sigma^{*}(\mathscr{C})$ and $M \in \sigma^{*}(\mathscr{D})$. Hence there exist $C \in \mathscr{C}$ and $D \in \mathscr{D}$ such that $M=\sigma(C)$ and $M=\sigma(D)$, besides $M=\sigma(C) \in \mathscr{C}$ and $M=\sigma(D) \in \mathscr{D}$. Hence $M \in \mathscr{C} \cap \mathscr{D}$, which implies that $M=\sigma(M) \in \sigma^{*}(\mathscr{C} \cap \mathscr{D})$. We conclude that $\sigma^{*}(\mathscr{C} \cap \mathscr{D})=\sigma^{*}(\mathscr{C}) \cap \sigma^{*}(\mathscr{D})$.

If $\mathbb{T} \in \sigma(R)$-tors, then we have that $\sigma^{*}(\overleftarrow{\sigma}(\mathbb{T})) \subseteq \mathbb{T}$. If $M \in \mathbb{T}$, then $\sigma(M) \in$ $\mathbb{T}$, this implies that $M \in \overleftarrow{\sigma}(\mathbb{T})$, from this we obtain $\sigma(M) \in \sigma^{*}(\overleftarrow{\sigma}(\mathbb{T}))$. As $M / \sigma(M) \in \mathbb{F}_{\sigma}$ we have that $M / \sigma(M) \in \sigma^{*}(\overleftarrow{\sigma}(\mathbb{T}))$ because $\mathbb{F}_{\sigma} \subseteq \sigma^{*}(\overleftarrow{\sigma}(\mathbb{T}))$. We get $M \in \sigma^{*}(\overleftarrow{\sigma}(\mathbb{T}))$, because $\mathbb{F}_{\sigma} \subseteq \sigma^{*}(\overleftarrow{\sigma}(\mathbb{T}))$ is closed under taking extensions. Hence $\mathbb{T} \subseteq \sigma^{*}(\overleftarrow{\sigma}(\mathbb{T}))$. We conclude that $\sigma^{*}(\overleftarrow{\sigma}(\mathbb{T}))=\mathbb{T}$

Analogously it can be shown that $\mathscr{C}=\overleftarrow{\sigma}\left(\sigma^{*}(\mathscr{C})\right)$ for all $\mathscr{C} \in R$-( $\sigma$-tors $)$. We conclude that $\sigma^{*}: R$ - $(\sigma$-tors $) \rightarrow \sigma(R)$-tors is a $\wedge$-isomorphism.

The following result is a generalization of Theorem 3.13.

Theorem 3.18. Let $I \leq R$ an ideal generated by a central idempotent in $R$ and take the preradical $\sigma$ defined as $\sigma(M)=I M . R$ - $(\sigma$-tors $)=\operatorname{Skel}\left(\mathscr{L}_{\left\{\leq_{\sigma}, \rightarrow_{\sigma}\right\}}\right)$ if and only if $I$ is a semiartinian ring.

Proof. From Remark $2.1 \sigma$ is an exact radical.

$(\Rightarrow)$ : Suppose that $R$-( $\sigma$-tors $)=\operatorname{Skel}\left(\mathscr{L}_{\left\{\leq_{\sigma}, \rightarrow_{\sigma}\right\}}\right)$. As $R$-Mod $\in R$ - $(\sigma$-tors $)$ we have that $\mathbb{T}_{\sigma}=\sigma^{*}(R$-Mod $) \in R$-tors and $\sigma^{*}(R$-Mod $)$ is the largest class in $\operatorname{Im}\left(\sigma^{*}\right)=\left\{\sigma^{*}(\mathbb{T}) \mid \mathbb{T} \in R\right.$-( $\sigma$-tors $\left.)\right\}$ (see Remark 2.2). Moreover, from Proposition 3.17, $\operatorname{Im}\left(\sigma^{*}\right)=I$-tors, this implies, by Proposition 3.17 that $I$-tors is a boolean lattice and $I$ is a semiartinian ring.

$(\Leftarrow)$ :) Let us take a semiartinian factor $I$ of $R$. We show that $R$ - $(\sigma$-tors $)=$ $\operatorname{Skel}\left(\mathscr{L}_{\left\{\leq_{\sigma}, \rightarrow_{\sigma}\right\}}\right)$. As $I$ is semiartinian, we have that for each $M \in R$-Mod that $\sigma(M)$ is semiartinian, this implies that $I$-tors $=\operatorname{Skel}\left(\mathscr{L}_{\{\leq, \rightarrow\}}^{I}\right)$. By Proposition 3.17 we have that $R$ - $(\sigma$-tors $)=\operatorname{Skel}\left(\mathscr{L}_{\left\{\leq_{\sigma}, \rightarrow_{\sigma}\right\}}\right)$.

3.1. $\sigma$-torsion theories. Since their introduction by Dickson [8] of torsion theories for Abelian categories, there have been defined several generalizations. We introduce a new extension of this concept. 
Definition 3.19. Let $\sigma$ be a preradical. Let us define the assignments $L_{\sigma}, R_{\sigma}$ : $\wp(R$-Mod $) \rightarrow \wp(R$-Mod $)$ as

(1) $L_{\sigma}(\mathscr{C})=\left\{M \in R\right.$-Mod $\left.\mid \operatorname{Hom}_{R}(\sigma(M), \sigma(N))=0, \forall N \in \mathscr{C}\right\}$,

(2) $R_{\sigma}(\mathscr{C})=\left\{N \in R\right.$-Mod $\left.\mid \operatorname{Hom}_{R}(\sigma(M), \sigma(N))=0, \forall M \in \mathscr{C}\right\}$.

It is immediate that for $\mathscr{C} \subseteq R$-Mod we have that $L_{\sigma}(\mathscr{C})=\overleftarrow{\sigma}\left(L\left(\sigma^{*}(\mathscr{C})\right)\right)$ and $R_{\sigma}(\mathscr{C})=\overleftarrow{\sigma}\left(R\left(\sigma^{*}(\mathscr{C})\right)\right.$

Notice that $L_{\sigma}$ and $R_{\sigma}$ are order reversing assignments and that $L_{\sigma} R_{\sigma}$ and $R_{\sigma} L_{\sigma}$ are closure operators. Besides, $L_{\sigma} R_{\sigma} L_{\sigma}=L_{\sigma}$ and $R_{\sigma} L_{\sigma} R_{\sigma}=R_{\sigma}$.

When $\sigma=1_{R \text {-Mod }}$ (see [14] Chap. VI), we have that

$$
\begin{aligned}
& L_{\sigma}(\mathscr{C})=L(\mathscr{C})=\left\{M \in R-\operatorname{Mod} \mid \operatorname{Hom}_{R}(M, N)=0, \forall N \in \mathscr{C}\right\}, \\
& R_{\sigma}(\mathscr{C})=R(\mathscr{C})=\left\{N \in R \text {-Mod } \mid \operatorname{Hom}_{R}(M, N)=0, \forall M \in \mathscr{C}\right\} .
\end{aligned}
$$

It is known that there exists a bijective correspondence between torsion theories and idempotent radicals, then for all $\mathscr{C} \subseteq R$-Mod we have that $L(\mathscr{C})=\mathbb{T}_{\tau}$ and $R(\mathscr{C})=\mathbb{F}_{\nu}$ for some idempotent radicals $\tau, \nu$, respectively.

Remark 3.20. Let $\sigma$ be a preradical and $M \in R$-Mod, then $M \in L_{\sigma}\left(R_{\sigma}(\{M\})\right)$ because

$$
\begin{gathered}
R_{\sigma}(\{M\})=\left\{N \in R \text {-Mod } \mid \operatorname{Hom}_{R}(\sigma(M), \sigma(N))=0\right\}, \text { and } \\
L_{\sigma}\left(R_{\sigma}(\{M\})\right)=\left\{L \in R \text {-Mod } \mid \operatorname{Hom}_{R}(\sigma(L), \sigma(N))=0, \forall N \in R_{\sigma}(\{M\})\right\} .
\end{gathered}
$$

If $\sigma$ is idempotent, then $\sigma(M) \in L_{\sigma}\left(R_{\sigma}(\{M\})\right)$ because for each $N \in R_{\sigma}(\{M\})$ we have that $\operatorname{Hom}_{R}(\sigma(\sigma(M)), \sigma(N))=\operatorname{Hom}_{R}(\sigma(M), \sigma(N))=0$.

Analogously, $N \in R_{\sigma}\left(L_{\sigma}(\{N\})\right)$ and if $\sigma$ is an idempotent preradical, then $\sigma(N) \in R_{\sigma}\left(L_{\sigma}(\{N\})\right)$.

Proposition 3.21. Let $\sigma$ be a preradical. For each $\mathscr{C} \subseteq R$-Mod, $R_{\sigma}(\mathscr{C}) \in$ $\mathscr{L}_{\{\leq \sigma, \Pi\}}$.

Proof. As $R_{\sigma}(\mathscr{C})=\overleftarrow{\sigma}\left(R\left(\sigma^{*}(\mathscr{C})\right)\right)$ and $R\left(\sigma^{*}(\mathscr{C})\right)$ is a torsion free class, then by Lemma 3.12 we obtain that $R_{\sigma}(\mathscr{C}) \in \mathscr{L}_{\{\leq \sigma\}}$.

Take $\left\{N_{\alpha}\right\}_{\alpha \in X} \subseteq R_{\sigma}(\mathscr{C})$ and $M \in \mathscr{C}$, then $\operatorname{Hom}_{R}\left(\sigma(M), \sigma\left(N_{\alpha}\right)\right)=0$ for each $\alpha \in X$, and $\sigma\left(\prod_{\alpha \in X} N_{\alpha}\right) \leq \prod_{\alpha \in X} \sigma\left(N_{\alpha}\right)$. Thus, we have a monomorphism $\operatorname{Hom}_{R}\left(\sigma(M), \sigma\left(\prod_{\alpha \in X} N_{\alpha}\right)\right) \rightarrow \operatorname{Hom}_{R}\left(\sigma(M), \prod_{\alpha \in X} \sigma\left(N_{\alpha}\right)\right)=0$ with

$$
\operatorname{Hom}_{R}\left(\sigma(M), \prod_{\alpha \in X} \sigma\left(N_{\alpha}\right)\right) \cong \prod_{\alpha \in X} \operatorname{Hom}_{R}\left(\sigma(M), \sigma\left(N_{\alpha}\right)\right)=0 .
$$

We conclude that $\operatorname{Hom}_{R}\left(\sigma(M), \sigma\left(\prod_{\alpha \in X} N_{\alpha}\right)\right)=0$, thus $\prod_{\alpha \in X} N_{\alpha} \in R_{\sigma}(\mathscr{C})$. Therefore $R_{\sigma}(\mathscr{C}) \in \mathscr{L}_{\left\{\leq_{\sigma}, \Pi\right\}}$. 
Proposition 3.22. Let $\sigma$ be a left exact preradical. For each $\mathscr{C} \subseteq R$-Mod, $R_{\sigma}(\mathscr{C}) \in$ $\mathscr{L}_{\{\leq \sigma, \Pi, e x t\}}$.

Proof. From Proposition 3.21 we have that $R_{\sigma}(\mathscr{C}) \in \mathscr{L}_{\left\{\leq_{\sigma}, \Pi\right\}}$. Let us see that it is also closed under extensions.

Let $0 \rightarrow N^{\prime} \rightarrow N \rightarrow N^{\prime \prime} \rightarrow 0$ be an exact sequence with $N^{\prime}, N^{\prime \prime} \in R_{\sigma}(\mathscr{C})$ and take $M \in \mathscr{C}$. Then $0 \rightarrow \sigma\left(N^{\prime}\right) \rightarrow \sigma(N) \rightarrow \sigma\left(N^{\prime \prime}\right)$ is an exact sequence, thus the sequence

$$
0 \rightarrow \operatorname{Hom}_{R}\left(\sigma(M), \sigma\left(N^{\prime}\right)\right) \rightarrow \operatorname{Hom}_{R}(\sigma(M), \sigma(N)) \rightarrow \operatorname{Hom}_{R}\left(\sigma(M), \sigma\left(N^{\prime \prime}\right)\right)
$$

is exact with $\operatorname{Hom}_{R}\left(\sigma(M), \sigma\left(N^{\prime}\right)\right)=0, \operatorname{Hom}_{R}\left(\sigma(M), \sigma\left(N^{\prime \prime}\right)\right)=0$. This implies that $\operatorname{Hom}_{R}(\sigma(M), \sigma(N))=0$. Hence $N \in R_{\sigma}(\mathscr{C})$. Therefore $R_{\sigma}(\mathscr{C}) \in \mathscr{L}_{\left\{\leq_{\sigma}, \Pi, e x t\right\}}$.

Proposition 3.23. Let $\sigma$ be an exact preradical. For each $\mathscr{C} \subseteq R$-Mod, $L_{\sigma}(\mathscr{C}) \in$ $R$-tors.

Proof. Assume that $\mathscr{C} \subseteq R$-Mod. As $L_{\sigma}(\mathscr{C})=\overleftarrow{\sigma}\left(L\left(\sigma^{*}(\mathscr{C})\right)\right)$ and $L\left(\sigma^{*}(\mathscr{C})\right) \in$ $R$-tors and $L\left(\sigma^{*}(\mathscr{C})\right) \supseteq \mathbb{F}_{\sigma}$ then the conclusion follows from Corollary 3.10.

Definition 3.24. Let $\sigma$ be an exact preradical. A $\sigma$-torsion theory is a pair of $R$-module classes $(\mathbb{T}, \mathbb{F})$ such that $\mathbb{T}=L_{\sigma}(\mathbb{F})$ and $\mathbb{F}=R_{\sigma}(\mathbb{T})$.

When $\sigma=1_{R \text {-Mod }}$ the $1_{R \text {-Mod-torsion theories are the usual torsion theories. }}$

\section{4. $\sigma$-retractable modules}

Definition 4.1. A left $R$-module $M$ is called retractable if for each $0 \neq N \leq M$ we have that $\operatorname{Hom}_{R}(M, N) \neq 0$.

In [10], it is proved that the class of mod-retractable commutative rings coincides with the class of commutative semiartinian rings. It is shown in [13] that every projective module over a right $V$-ring is retractable.

We mention some examples: free modules and semisimple modules are retractable. Any direct sum of modules of the form $\mathbb{Z}_{p^{i}}$ is retractable, where $p$ is a prime number. The $\mathbb{Z}$-module $\mathbb{Z}_{p^{\infty}}$ is not retractable.

Let us recall that there is a one to one correspondence between the class of left exact radicals and the class of hereditary torsion theories (See [14] Chap. VI).

The following theorem is proved in [10], we include a proof as an illustration.

Theorem 4.2. ([10]) $R$-tors $=R$-TORS if and only if each $R$-module is retractable. 
Proof. $(\Rightarrow)$ : Assume that $R$-tors $=R$-TORS and take $\mathbb{T} \in R$-tors. Take $M \in \mathbb{T}$ and $0 \neq N \leq M$.

Let $\mathbb{T}$ be the least torsion containing $M$, then $N \in \mathbb{T}$ implies that $N \notin \mathbb{F}$ hence $\operatorname{Hom}_{R}(M, N) \neq 0$. We conclude that $M$ is retractable.

$(\Leftarrow)$ : Suppose that each $R$-module is retractable and take $\mathbb{T}_{\sigma} \in R$-TORS. Take $M \in \mathbb{T}_{\sigma}$ and $N \leq M$

If $N \notin \mathbb{T}_{\sigma}$, let us take the exact sequence $0 \rightarrow \sigma(N) \rightarrow N \rightarrow N / \sigma(N) \rightarrow 0$. In the diagram

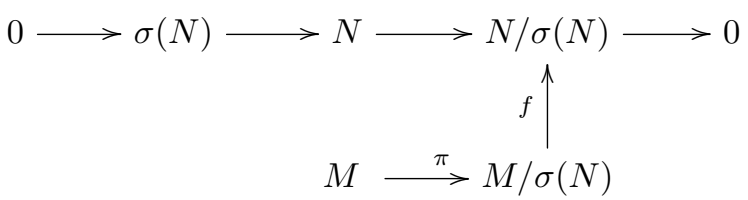

we have that $M / \sigma(N) \in \mathbb{T}_{\sigma}$ and $N / \sigma(N) \leq M / \sigma(N)$. Then there exists a nonzero $f: M / \sigma(N) \rightarrow N / \sigma(N)$. This implies that $f(M / \sigma(N)) \in \mathbb{T}_{\sigma}$.

Let us take $N^{\prime} / \sigma(N)=f(M / \sigma(N))$. We have the exact sequence

$$
0 \rightarrow \sigma(N) \rightarrow N^{\prime} \rightarrow N^{\prime} / \sigma(N) \rightarrow 0
$$

with $\sigma(N), N^{\prime} / \sigma(N) \in \mathbb{T}_{\sigma}$. From this, we have that $N^{\prime} \in \mathbb{T}_{\sigma}$ con $N^{\prime} \leq N$. This implies that $N^{\prime}=\sigma\left(N^{\prime}\right) \leq \sigma(N)$, thus $N^{\prime}=\sigma(N)$ (because $\sigma$ is left exact). It follows that $f(M / \sigma(N))=N^{\prime} / \sigma(N)=0$, contradicting that $f \neq 0$. Hence $\sigma(N)=N$

Hence $N \in \mathbb{T}_{\sigma}$, and $\mathbb{T}_{\sigma}$ is closed under taking submodules.

Definition 4.3. Let $\sigma$ be a preradical. An $R$-module $M$ is called left $\sigma$-retractable if for each $N \leq M$ with $\sigma(N) \neq 0$, one has that $\operatorname{Hom}_{R}(M, \sigma(N)) \neq 0$. A ring $R$ will be called $\sigma$-(R-Mod)-retractable if each $R$-module is $\sigma$-retractable.

Remark 4.4. Notice that each retractable $R$-module is $\sigma$-retractable, but a non retractable $R$-module $M$ can be $\sigma$-retractable for some $\sigma \in R$-pr.

As an example, let $t \in \mathbb{Z}$-pr denote the torsion functor and take the $\mathbb{Z}$-module $\mathbb{Q}$. For each $N \leq \mathbb{Q}$ we have that $t(N)=N \cap t(\mathbb{Q})=N \cap 0=0$. Hence by vacuity, $\mathbb{Q}$ is $t$-retractable, but it is not retractable, because for $\frac{a}{b} \mathbb{Z} \hookrightarrow \mathbb{Q}$, with $a, b \in \mathbb{Z}$ and $a, b \neq 0$ it happens that $\operatorname{Hom}_{\mathbb{Z}}\left(\mathbb{Q}, \frac{a}{b} \mathbb{Z}\right)=0$.

Remark 4.5. For each preradical $\sigma$, each $M \in R$-Mod and $N \leq M$, if $\sigma(M / N)=$ 0 , then $\sigma(M) \leq N$ (see [5] Prop. I.1.1).

Theorem 4.6. Let $\sigma$ be a left exact preradical. If each $R$-module is $\sigma$-retractable, then $R$ - $(\sigma$-tors $)=R$ - $(\sigma$-TORS $)$. 
Proof. Let us assume that $\mathbb{T} \in R$-( $\sigma$-TORS $)$ and $M \in \mathbb{T}$. Assume also that $0 \neq \sigma(N) \leq N \leq M$. We will show that $\sigma(N) \in \mathbb{T}$.

If there exists a nonzero homomorphism $f: M \rightarrow \sigma(N)$, then $\sigma(f(M)) \in \mathbb{T}$, so there exists a nonzero submodule $L$ of $\sigma(N)$ with $L \in \mathbb{T}$. Let us denote $\mathcal{U}=$ $\{L \leq \sigma(N) \mid L \in \mathbb{T}\}$. We have that $\bigoplus_{L \in \mathcal{U}} L \in \mathbb{T}$ and that there is an epimorphism $\bigoplus_{L \in \mathcal{U}} L \rightarrow \sum_{L \in \mathcal{U}} L$. Hence $\sigma\left(\sum_{L \in \mathcal{U}} L\right) \in \mathbb{T}$ and $\sigma\left(\sum_{L \in \mathcal{U}} L\right)$ is the largest submodule of $\sigma(N)$ belonging to $\mathbb{T}$. Let us denote $V=\sigma\left(\sum_{L \in \mathcal{U}} L\right)$.

We have that $\sigma(N) / V \leq N / V \leq M / V$. We have two cases: $\sigma(\sigma(N) / V)=0$ and $\sigma(\sigma(N) / V) \neq 0$.

In the former case, $\sigma(\sigma(N) / V)=0$, we have that $\sigma(N)=\sigma(\sigma(N)) \leq V$ (see Remark 4.5), this implies that $\sigma(N)=V \in \mathbb{T}$.

If $\sigma(\sigma(N) / V) \neq 0$, then $\sigma(M / V) \neq 0$ and we have that there exists a nonzero homomorphism $h: M / V \rightarrow \sigma(\sigma(N) / V)$. From this, we have that $h(M / V)=$ $\sigma(h(M / V)) \in \mathbb{T}$. Taking $U \leq \sigma(N)$ such that $U / V=h(M / V)$, we obtain the exact sequence $0 \longrightarrow V \longrightarrow U \longrightarrow U / V \longrightarrow 0$ which has $V, U / V \in \mathbb{T}$. We get that $U \in \mathbb{T}$.

Then $U$ is a submodule of $\sigma(N)$ such that $U \in \mathbb{T}$, this implies that $U \leq V$. We conclude that $U=V$ and from this, that $0=U / V=h(M / V)$, a contradiction. Hence $\sigma(N)=V \in \mathbb{T}$ and $\mathbb{T}$ is $\sigma$-hereditary.

Proposition 4.7. Let $\sigma$ be a left exact preradical. If $R$ - $(\sigma$-TORS $)=R$ - $(\sigma$-tors $)$, then for each $\mathbb{T} \in R$-( $\sigma$-TORS $), \sigma^{*}(\mathbb{T})$ is a hereditary torsion class.

Proof. Take $\mathbb{T} \in R$-( $\sigma$-TORS $), M \in \sigma^{*}(\mathbb{T})$ and $N \leq M$. We will show that $N \in \sigma^{*}(\mathbb{T})$.

By hypothesis there exists $L \in \mathbb{T}$ such that $\sigma(L)=M$. As $\sigma(L) \in \mathbb{T}, N \leq M=$ $\sigma(L)$ and $\sigma$ is left exact, we have that $N=\sigma(N) \in \mathbb{T}$, because it is a $\sigma$-hereditary class, this implies that $N=\sigma(N) \in \sigma^{*}(\mathbb{T})$. Hence $\sigma^{*}(\mathbb{T})$ is hereditary.

Proposition 4.8. Let $\sigma$ be an exact preradical. If $R$ - $(\sigma$-TORS $)=R$-( $\sigma$-tors $)$ then each $R$-module $M \in \mathbb{T}_{\sigma}$ is $\sigma$-retractable.

Proof. Let us assume that $R$ - $(\sigma$-TORS $)=R$-( $\sigma$-tors $)$, take $M \in \mathbb{T}_{\sigma}$ and $N \leq M$. We show that $M$ is a $\sigma$-retractable module. Let us suppose that $\operatorname{Hom}_{R}(M, \sigma(N))=$ 0 . We will show that $\sigma(N)=0$.

From Proposition 3.23 we have that $R$ - $(\sigma$-TORS $)=\left\{L_{\sigma}(\mathscr{C}) \mid \mathscr{C} \subseteq R\right.$-Mod $\}$. Then $L_{\sigma}\left(R_{\sigma}(\{M\})\right) \in R$ - $\left(\sigma\right.$-TORS) and $\sigma(M)=M \in L_{\sigma}\left(R_{\sigma}(\{M\})\right)$ (see Remark 3.20). As $L_{\sigma}\left(R_{\sigma}(\{M\})\right)$ is a $\sigma$-hereditary class, we have $\sigma(N) \in L_{\sigma}\left(R_{\sigma}(\{M\})\right)$, and $\operatorname{Hom}_{R}(\sigma(M), \sigma(\sigma(N)))=\operatorname{Hom}_{R}(\sigma(M), \sigma(N))=0$ implies that $\sigma(N) \in R_{\sigma}(\{M\})$. 
This means that $\sigma(N) \in L_{\sigma}\left(R_{\sigma}(\{M\})\right) \cap R_{\sigma}(\{M\})=\mathbb{F}_{\sigma}$, from this $\sigma(N)=$ $\sigma(\sigma(N))=0$ follows. It follows that $M$ is a $\sigma$-retractable module.

Example 4.9. Let $I \leq R$ be a two sided pure ideal and let $t\left(\alpha_{I}^{R}\right) \in R$-pr be defined by $t\left(\alpha_{I}^{R}\right)(M)=\{m \in M \mid I m=0\}$, the annihilator of $I$ on $M$. Notice that there is a natural isomorphism $t\left(\alpha_{I}^{R}\right) \cong R / I \otimes_{R}-$. Then $t\left(\alpha_{I}^{R}\right)$ is an exact preradical $(R / I$ is flat). Thus, for all $M \in R$-Mod we have that $t\left(\alpha_{I}^{R}\right)(M) \cong M / I M$. Notice that

(1) $t\left(\alpha_{I}^{R}\right)(M)=0 \Leftrightarrow M=I M$.

(2) $t\left(\alpha_{I}^{R}\right)(M)=M \Leftrightarrow I M=0 \Leftrightarrow M \in R / I$-Mod.

$M$ is a $t\left(\alpha_{I}^{R}\right)$-retractable module if for all $N \leq M$ with $I N \neq N$, we have that $\operatorname{Hom}_{R}(M, N / I N) \neq 0$. By Proposition 4.6, if each $R$-module $M$ is $t\left(\alpha_{I}^{R}\right)$ retractable, then $R$ - $\left(t\left(\alpha_{I}^{R}\right)\right.$-TORS $)=R$ - $\left(t\left(\alpha_{I}^{R}\right)\right.$-tors $)$. By Proposition 4.8, if $R$ $\left(t\left(\alpha_{I}^{R}\right)\right.$-TORS $)=R-\left(t\left(\alpha_{I}^{R}\right)\right.$-tors $)$, then each $R / I$-module is $t\left(\alpha_{I}^{R}\right)$-retractable.

Example 4.10. If $R=S \times T$ with $S$ and $T$ two rings. Define $\sigma \in R$-pr by $\sigma(M)=e M$, where $e=(1,0)$. Then $R$ - $(\sigma$-TORS $)=R$ - $(\sigma$-tors $)$ if and only if each $R$-module $\sigma$-retractable, which is equivalent to each $S$-module be retractable and $S$-TORS $=S$-tors

\section{5. $\sigma$-open and $\sigma$-stable classes}

The big lattice of $\sigma$-open classes is denoted by $\mathscr{L}_{\left\{\leq_{\sigma}, \rightarrow_{\sigma}\right\}}$.

Remark 5.1. If $\sigma$ is an idempotent preradical, then for each $M, N \in R$-Mod and each epimorphism $g: \sigma(M) \rightarrow N$ we have that $\sigma(N)=N$, because

$$
N=g(\sigma(M))=g(\sigma(\sigma(M))) \leq \sigma(N) \leq N .
$$

Proposition 5.2. Let $\sigma$ be a cohereditary idempotent preradical. There is an assignment $\rho_{\rightarrow_{\sigma}}: \mathscr{L}_{\left\{\leq_{\sigma}, \rightarrow_{\sigma}\right\}} \rightarrow R$-( $\sigma$-TORS $)$ defined by

$$
\rho_{\rightarrow \sigma}(\mathscr{C})=\{M \in R-\operatorname{Mod} \mid \forall M \rightarrow L,(\sigma(L) \in \mathscr{C} \Rightarrow \sigma(L)=0)\} \cup \mathbb{F}_{\sigma} .
$$

Proof. If $\mathscr{C} \in \mathscr{L}_{\left\{\leq_{\sigma} \rightarrow \rightarrow_{\sigma}\right\}}$, we will show that $\rho_{\rightarrow_{\sigma}}(\mathscr{C}) \in R$-( $\sigma$-TORS). To get started, we show that if $M \in \rho_{\rightarrow_{\sigma}}(\mathscr{C})$, then $\sigma(M) \in \rho_{\rightarrow \sigma}(\mathscr{C})$.

Let us take $M \in \rho_{\rightarrow_{\sigma}}(\mathscr{C})$ and $g: M \rightarrow N$. We will show $\sigma(N) \in \rho_{\rightarrow \sigma}(\mathscr{C})$. Further take $f: \sigma(N) \rightarrow L$ and let us suppose that $\sigma(L) \in \mathscr{C}$ and $\sigma(L) \neq 0$. We have that $\sigma(L)=L$ (see Remark 5.1). We have the following commutative diagram: 


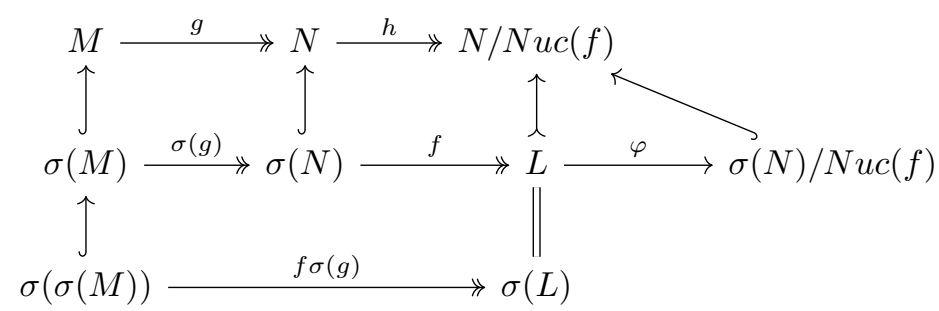

where $\varphi$ is an isomorphism. As $\sigma$ is cohereditary and $\bar{f}$ is an epimorphism then $\sigma(\bar{f}): \sigma(N) \rightarrow \sigma(N / N u c(f))$ is an epimorphism, $\sigma(N / N u c(f))=\sigma(N) / N u c(f) \cong$ $L \in \mathscr{C}$ and $0 \neq L \cong \sigma(N / N u c(f))$. This is a contradiction since $\bar{f} \circ g: M \rightarrow$ $N / N u c(f)$ is an epimorphism with $M \in \rho_{\rightarrow \sigma}(\mathscr{C})$. Therefore $\sigma(L) \in \mathscr{C}$ implies $\sigma(L)=0$, then $\sigma(N) \in \rho_{\rightarrow \sigma}(\mathscr{C})$.

Now, let $0 \longrightarrow M^{\prime} \stackrel{f}{\longrightarrow} M \stackrel{g}{\longrightarrow} M^{\prime \prime} \longrightarrow 0$ be an exact sequence with $M^{\prime}, M^{\prime \prime} \in$ $\rho_{\rightarrow_{\sigma}}(\mathscr{C})$. We are going to show that $M \in \rho_{\rightarrow_{\sigma}}(\mathscr{C})$. As $\sigma$ is idempotent and cohereditary, then it is an idempotent radical and $\mathbb{F}_{\sigma}$ is closed under taking extensions.

If $h: M \rightarrow L$ is an epimorphism with $\sigma(L) \neq 0$, we are going to show that $\sigma(L) \notin \mathscr{C}$. We have the following commutative diagram:

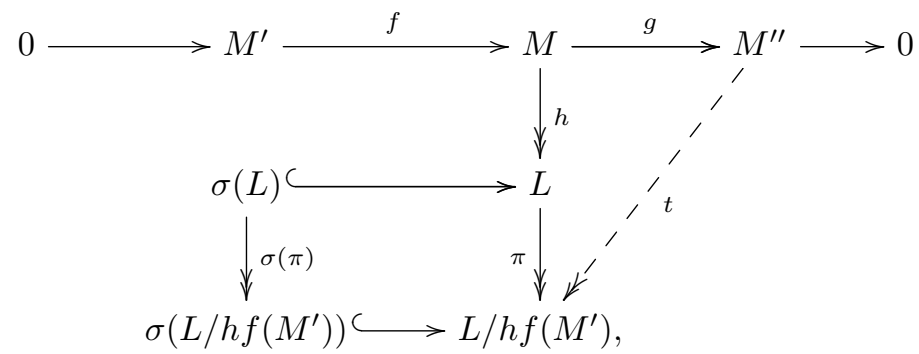

where $\pi$ denotes the natural epimorphism, and $t: M^{\prime \prime} \rightarrow L / h f\left(M^{\prime}\right)$ is an epimorphism. Notice now that $h f: M^{\prime} \rightarrow h f\left(M^{\prime}\right)$ is an epimorphism with $M^{\prime} \in \rho_{\rightarrow_{\sigma}}(\mathscr{C})$.

Let us first consider the case where $M^{\prime} \notin \mathbb{F}_{\sigma}$ and $M^{\prime \prime} \notin \mathbb{F}_{\sigma}$. If $0 \neq \sigma(L) \in \mathscr{C}$, we have the exact sequence

$$
0 \rightarrow h f\left(M^{\prime}\right) \rightarrow L \rightarrow L / h f\left(M^{\prime}\right) \rightarrow 0,
$$

from which we obtain the exact sequence

$$
\sigma\left(h f\left(M^{\prime}\right)\right) \rightarrow \sigma(L) \rightarrow \sigma\left(L / h f\left(M^{\prime}\right)\right) \rightarrow 0 .
$$

Since $\sigma(L) \in \mathscr{C}, \sigma$ is idempotent and $\mathscr{C} \in \mathscr{L}_{\{\rightarrow \sigma\}}$, then $\sigma\left(L / h f\left(M^{\prime}\right)\right) \in \mathscr{C}$. But since $L / h f\left(M^{\prime}\right)$ is a quotient of $M^{\prime \prime}$, then $\sigma\left(L / h f\left(M^{\prime}\right)\right)$ has to be 0 . Thus $\sigma\left(h f\left(M^{\prime}\right)\right)=\sigma(L)$, contradicting than $M^{\prime} \in \rho_{\rightarrow_{\sigma}}(\mathscr{C})$.

Now consider the case $M^{\prime} \in \mathbb{F}_{\sigma}$ and $M^{\prime \prime} \notin \mathbb{F}_{\sigma}$. As in the previous case, we obtain $\sigma\left(L / h f\left(M^{\prime}\right)\right)=\sigma(L) \neq 0$, in contradiction to the hypothesis that $M^{\prime} \in \mathbb{F}_{\sigma}$ 
If $M^{\prime} \notin \mathbb{F}_{\sigma}$ and $M^{\prime \prime} \in \mathbb{F}_{\sigma}$ then $\sigma\left(L / h f\left(M^{\prime}\right)\right)=0$, so $\sigma(L) \subseteq h f\left(M^{\prime}\right) \subseteq L$. Using that $\sigma$ is idempotent, we obtain that $\sigma(L)=\sigma\left(h f\left(M^{\prime}\right)\right)=0$. This contradicts the hypothesis.

Finally, if $M^{\prime}, M^{\prime \prime} \in \mathbb{F}_{\sigma}$, as in the previous case, we have $\sigma(L)=\sigma\left(h f\left(M^{\prime}\right)\right)=0$, since $M^{\prime} \in \mathbb{F}_{\sigma}$. This is a contradiction. We conclude that $M \in \rho_{\rightarrow_{\sigma}}(\mathscr{C})$.

Now, take a family $\left\{M_{\alpha}\right\}_{\alpha \in X} \in \rho_{\rightarrow_{\sigma}}(\mathscr{C})$. We are going to show $\bigoplus_{\alpha \in X} M_{\alpha} \in$ $\rho_{\rightarrow \sigma}(\mathscr{C})$. Let us suppose there is an epimorphism $\bigoplus_{\alpha \in X} M_{\alpha} \rightarrow L$ with $\sigma(L) \in \mathscr{C}$ and $\sigma(L) \neq 0$.

Note that we always have an epimorphism $h: \bigoplus_{l \in L} R l \rightarrow L$, then $\sigma(h): \bigoplus_{l \in L} \sigma(R l) \rightarrow$ $\sigma(L)$ is an epimorphism. As $\sigma(L) \neq 0$, there exists $0 \neq l \in \sigma(L)$ such that $\sigma(R l) \neq 0$, furthermore $\sigma(R l) \in \mathscr{C}$.

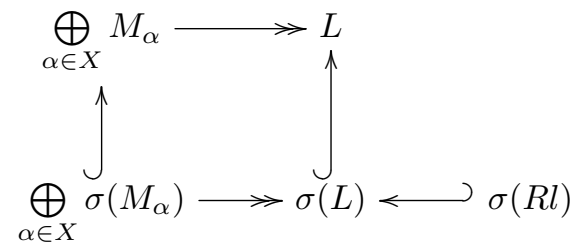

We have that $l=m_{\alpha_{1}}+m_{\alpha_{2}}+\cdots+m_{\alpha_{k}}$ with $m_{\alpha_{j}} \in M_{\alpha_{j}}$ con $1 \leq j \leq k$. Then for each $j$ there exists an epimorphism $M_{\alpha_{j}} \rightarrow R m_{j}$ with $\sigma\left(R m_{j}\right) \in \mathscr{C}$, this implies $\sigma\left(R m_{j}\right)=0$ for each $j$. Then $\sigma(L)=0$ (because of the epimorphism $\left.0=\bigoplus_{j=1}^{k} \sigma\left(R m_{\alpha_{j}}\right)=\sigma\left(\bigoplus_{j=1}^{k} R m_{\alpha_{j}}\right) \rightarrow \sigma(L)\right)$, contradicting that $\sigma(L) \neq 0$. Hence $\sigma(L) \in \mathscr{C}$ implies that $\sigma(L)=0$. We conclude that $\bigoplus_{\alpha \in X} M_{\alpha} \in \rho_{\rightarrow_{\sigma}}(\mathscr{C})$.

Proposition 5.3. Let $\sigma$ be an idempotent preradical. We have the assignment $\rho_{\rightarrow_{\sigma}}: \mathscr{L}_{\left\{\leq_{\sigma}, \sigma E\right\}} \rightarrow \mathscr{L}_{\left\{\leq_{\sigma}\right\}} \cdot$

Proof. Take $M \in \rho_{\rightarrow_{\sigma}}(\mathscr{C})$ and $N \leq M$. We prove that $\sigma(N) \in \rho_{\rightarrow_{\sigma}}(\mathscr{C})$. If $g: \sigma(N) \rightarrow L$ is an epimorphism, with $\sigma(L) \in \mathscr{C}$ and $\sigma(L) \neq 0$, then we have that $\sigma(L)=L$ (see Remark 5.1). We have the following commutative diagram:

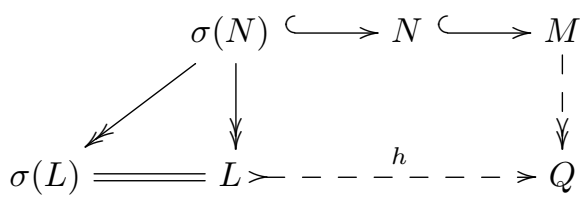

Let $Q^{\prime} \leq Q$ be a pseudocomplement of $h(L) \leq Q$, then $h(L)+Q^{\prime} \leq e$ and there exists an essential monomorphism $L \stackrel{\text { ess }}{\longmapsto} Q / Q^{\prime}$, then there also exists a monomorphism $Q / Q^{\prime} \longmapsto E(L)$. 


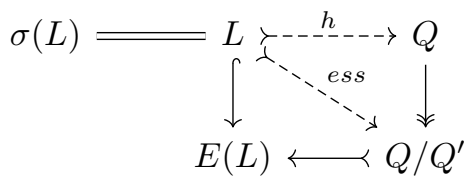

Thus, we have that $E(L) \cong E\left(Q / Q^{\prime}\right)$. As $\sigma(L) \in \mathscr{C}$, it follows that $\sigma\left(E\left(Q / Q^{\prime}\right)\right) \in \mathscr{C}$ because $\sigma\left(E\left(Q / Q^{\prime}\right)\right) \cong \sigma(E(L))=\sigma(E(\sigma(L)))$. As $\sigma\left(Q / Q^{\prime}\right) \leq$ $\sigma\left(E\left(Q / Q^{\prime}\right)\right)$, we have that $\sigma\left(Q / Q^{\prime}\right)=\sigma\left(\sigma\left(Q / Q^{\prime}\right)\right) \in \mathscr{C}$, thus as $M \in \rho_{\rightarrow \sigma}(\mathscr{C})$ and $\sigma\left(Q / Q^{\prime}\right) \in \mathscr{C}$ we have that $\sigma\left(Q / Q^{\prime}\right)=0$ because $M \rightarrow Q / Q^{\prime}$ is an epimorphism. Hence $\sigma\left(Q / Q^{\prime}\right)=0$ implies that $\sigma(Q) \leq Q^{\prime}$. As $h(L) \leq \sigma(Q)$, we have that $h(L) \cap Q^{\prime}=0$ implies that $L \cong h(L)=0$, contradiction. Hence $\sigma(L) \in \mathscr{C}$ implies $\sigma(L)=0$. We conclude that $\sigma(N) \in \rho_{\rightarrow_{\sigma}}(\mathscr{C})$ and $\rho_{\rightarrow_{\sigma}}(\mathscr{C}) \in \mathscr{L}_{\left\{\leq_{\sigma}\right\}}$.

From Propositions 5.2 and 5.3 we have the following result:

Corollary 5.4. If $\sigma$ is a cohereditary preradical, we have the following assignments.

(1) $\rho_{\rightarrow_{\sigma}}: \mathscr{L}_{\left\{\leq_{\sigma}, \rightarrow_{\sigma}\right\}} \rightarrow R$-( $\sigma$-TORS $)$

(2) $\rho_{\rightarrow_{\sigma}}: \mathscr{L}_{\left\{\leq_{\sigma}, \rightarrow_{\sigma}, \sigma E\right\}} \rightarrow R$-( $\sigma$-tors).

(3) If besides $\sigma$ is a left exact stable preradical, then we have the assignment $\rho_{\rightarrow \sigma}: \sigma$-(R-Nat $) \rightarrow R$-( $\sigma$-tors $)$.

In [12] is given an assignment between $R$-Nat and $R$-tors. From the preceding

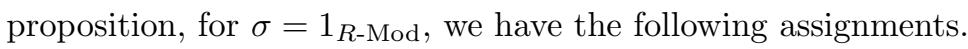

Corollary 5.5. There exist assignments:

(1) $\rho_{\rightarrow}: \mathscr{L}_{\{\leq, \rightarrow\}} \rightarrow R$-TORS.

(2) $\rho_{\rightarrow}: \mathscr{L}_{\{\leq, \rightarrow, E\}} \rightarrow R$-tors.

(3) $\rho_{\rightarrow}: R$-Nat $\rightarrow R$-tors.

(4) Furthermore, we have the commutative diagram:

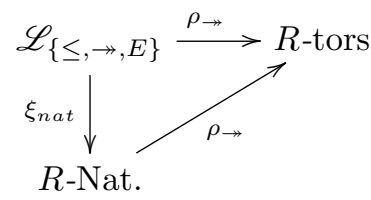

Recall that we denote $R$-jtors the collection of all hereditary jansian torsion theories, i.e., the collection of hereditary torsion classes closed under taking products. Notice that $R$ is a left perfect ring if and only if every hereditary right torsion class is closed under taking products and it is generated by a family of right simple modules (notice the change of side). Thus, it could happen that $R$-tors $\neq R$-jtors even if $R$ is left perfect. 
Proposition 5.6. If $R$ is a left perfect ring, then we have the following assignment $\rho_{\leq}: \mathscr{L}_{\{\leq, \rightarrow, P\}} \rightarrow R$-jtors, defined by

$$
\rho_{\leq}(\mathscr{C})=\{M \in R-\operatorname{Mod} \mid L \longmapsto M, L \in \mathscr{C} \Rightarrow L=0\} .
$$

Proof. Let $\mathscr{C} \in \mathscr{L}_{\{\leq, \rightarrow, P\}}$, we prove that $\rho_{\leq}(\mathscr{C}) \in R$-jtors. Further, take $M \in$ $\rho_{\leq}(\mathscr{C})$ and $N \leq M$. We prove that $N \in \rho_{\leq}(\mathscr{C})$.

Let us assume that there exists a monomorphism $L \longmapsto N$ with $L \in \mathscr{C}$ and $L \neq 0$. Then we have that the composition $L \longmapsto N \succ M$ is a monomorphism with $M \in \rho_{\leq}(\mathscr{C})$ and $L \in \mathscr{C}$. This implies that $L=0$, a contradiction. It follows that $N \in \rho_{\leq}(\mathscr{C})$.

Now, take $M \in \rho_{\leq}(\mathscr{C})$ and $g: M \rightarrow N$. We are going to show that $N \in \rho_{\leq}(\mathscr{C})$. Suppose that there exists $f: L \longmapsto N$ with $L \in \mathscr{C}$ and $L \neq 0$. Suppose there is a projective cover $f: P(L) \rightarrow L$ of $L$, thus $P(L) \in \mathscr{C}$. We have the following commutative diagram

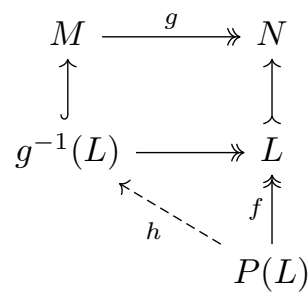

There is a homomorphism $0 \neq h: P(L) \rightarrow g^{-1}(L)$ making the diagram commutative, and we have that $0 \neq h(P(L)) \in \mathscr{C}$, as $h(P(L)) \leq M$ we have that $h(P(L))=0$, a contradiction. Hence $P(L)=0$, thus $L=0$, contradicting that $L \neq 0$. Hence $L \in \mathscr{C}$ implies that $L=0$. It follows that $N \in \rho_{\leq}(\mathscr{C})$.

Now, let $0 \rightarrow M^{\prime} \rightarrow M \rightarrow M^{\prime \prime} \rightarrow 0$ be an exact sequence with $M^{\prime}, M^{\prime \prime} \in \rho_{\leq}(\mathscr{C})$. We are going to show that $M \in \rho_{\leq}(\mathscr{C})$. Let us take a monomorphism $L \longmapsto M$ with $L \in \mathscr{C}$ and $L \neq 0$.

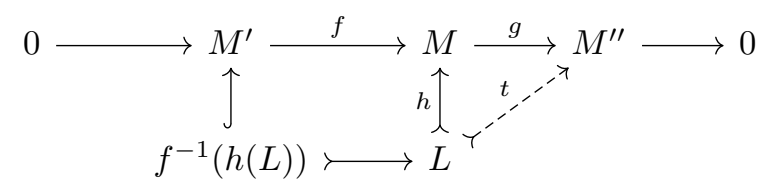

As $M^{\prime} \in \rho_{\leq}(\mathscr{C})$ and $f^{-1}(h(L)) \leq M^{\prime}$, then $f^{-1}(h(L))=0$, because $f^{-1}(h(L)) \cong$ $L \in \mathscr{C}$. Then

$$
f^{-1}\left(f\left(M^{\prime}\right) \cap h(L)\right)=M^{\prime} \cap f^{-1}(h(L))=M^{\prime} \cap 0=0 .
$$

Hence there exists a monomorphism $t: L \longmapsto M^{\prime \prime}$ which implies that $L=0$, a contradiction. We conclude that $L \in \mathscr{C}$ implies that $L=0$, thus $M \in \rho_{\leq}(\mathscr{C})$. 
Finally, suppose that $\left\{M_{\alpha}\right\}_{\alpha \in X} \subseteq \rho_{\leq}(\mathscr{C})$. We are going to show that $\prod_{\alpha \in X} M_{\alpha} \in$ $\rho_{\leq}(\mathscr{C})$. Assume that $f: L \longmapsto \prod_{\alpha \in X} M_{\alpha}$ is a monomorphism with $L \in \mathscr{C}$ and $L \neq 0$. Take $\pi_{\beta}: \prod_{\alpha \in X} M_{\alpha} \rightarrow M_{\beta}$ such that $\pi_{\beta} \circ f \neq 0$, thus $0 \neq \pi_{\beta}(f(L)) \leq M_{\beta}$.

If $0 \neq R l \leq L$, then $R l \in \mathscr{C}$. Let us take $0 \neq l=m_{\alpha_{1}}+\cdots+m_{\alpha_{k}}$ with the least possible $k$. Then there exists a monomorphism $R l \longmapsto M_{\alpha_{1}}$ with $R m_{\alpha_{1}} \in \mathscr{C}$ (see Proposition 6 in [6]). This implies that $R_{\alpha_{1}}=0$, and thus $m_{\alpha_{1}}=0$, a contradiction to the choice of $k$. Hence $L \in \mathscr{C}$ and $L=0$. We conclude that $\prod_{\alpha \in X} M_{\alpha} \in \rho_{\leq}(\mathscr{C})$. Hence $\rho_{\leq}(\mathscr{C}) \in R$-tors $\cap \mathscr{L}_{\{\Pi\}}$.

Let $\sigma$ be a preradical, we have the assignment

$$
\rho_{\leq_{\sigma}}(\mathscr{C})=\{M \in R \text {-Mod } \mid L \longmapsto M, \sigma(L) \in \mathscr{C} \Rightarrow \sigma(L)=0\}
$$

Notice that $\mathbb{F}_{\sigma} \subseteq \rho_{\leq \sigma}(\mathscr{C})$. We denote $R$ - $(\sigma$-jtors $):=R$ - $(\sigma$-tors $) \cap \mathscr{L}_{\{\Pi\}}$.

Proposition 5.7. Let $R$ be a left perfect ring. Let $\sigma$ be an exact and costable preradical. We have an assignment $\rho_{\leq_{\sigma}}: \mathscr{L}_{\left\{\leq_{\sigma}, \rightarrow_{\sigma}, \sigma P\right\}} \rightarrow R$-( $\sigma$-jtors).

Proof. Let $\mathscr{C} \in \mathscr{L}_{\left\{\leq_{\sigma}, \rightarrow_{\sigma}, \sigma P\right\}}$. We are going show that $\rho_{\leq_{\sigma}}(\mathscr{C}) \in R$-( $\sigma$-jtors $)$. Take $M \in \rho_{\leq_{\sigma}}(\mathscr{C})$ and $N \leq M$. We are going to show that $\sigma(N) \in \rho_{\leq_{\sigma}}(\mathscr{C})$.

If $L \longmapsto \sigma(N)$ is a monomorphism with $\sigma(L) \neq 0$, then as $\sigma(M) \in \rho_{\leq_{\sigma}}(\mathscr{C})$ and the composition $\sigma(L) \longmapsto \sigma(N) \longmapsto \sigma(M)$ is a monomorphism with $\sigma(\sigma(L))=\sigma(L) \neq 0$, we have that $\sigma(L)=\sigma(\sigma(L)) \notin \mathscr{C}$. Hence $\sigma(N) \in \rho_{\leq_{\sigma}}(\mathscr{C})$.

Now, take $M \in \rho_{\leq_{\sigma}}(\mathscr{C})$ and $g: M \rightarrow N$. We are going to show that $\sigma(N) \in$ $\rho_{\leq_{\sigma}}(\mathscr{C})$. Let us suppose that $f: L \longmapsto \sigma(N)$ is a monomorphism with $\sigma(L) \in \mathscr{C}$ and $\sigma(L) \neq 0$. Notice that $\sigma(L) \cong \sigma(f(L))=f(L) \cap \sigma(N)=f(L) \cong L$. Let $h$ : $P(L) \rightarrow L$ be a projective cover of $L$. We have that $h(\sigma(P(L)))=\sigma(L) \neq 0$, from this it follows $\sigma(P(L)) \in \mathscr{C}$ and $\sigma(P(L)) \neq 0$. We have the following commutative diagram:

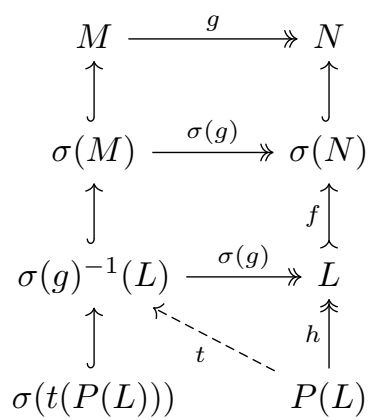

As $\sigma(P(L)) \neq 0$ we have that $\sigma(t(P(L))) \neq 0$ and $\sigma(t(P(L))) \in \mathscr{C}$, because $\sigma(t): \sigma(P(L)) \rightarrow \sigma(t(P(L)))$ is an epimorphism. As $\sigma(M) \in \rho_{\leq_{\sigma}}(\mathscr{C})$ we have that 
$\sigma(t(P(L))) \notin \mathscr{C}$, a contradiction. Hence, $\sigma(t(P(L)))=0$, thus $\sigma(t)=0$. This implies that $\sigma(P(L))=0$, a contradiction.

Then we have that $\sigma(L) \in \mathscr{C}$ implies that $\sigma(L)=0$. We conclude that $\sigma(N) \in$ $\rho_{\leq \sigma}(\mathscr{C})$.

Now, let $0 \rightarrow M^{\prime} \stackrel{f}{\longrightarrow} M \stackrel{g}{\longrightarrow} M^{\prime \prime} \rightarrow 0$ be an exact sequence with $M^{\prime}, M^{\prime \prime} \in$ $\rho_{\leq_{\sigma}}(\mathscr{C})$. We show that $M \in \rho_{\leq_{\sigma}}(\mathscr{C})$.

Let $h: L \longmapsto M$ be a monomorphism with $\sigma(L) \neq 0$. We have the following commutative diagram.

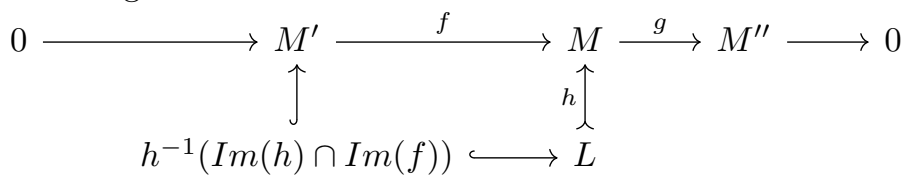

If $\operatorname{Im}(h) \cap \operatorname{Im}(f)=0$, then there exists a monomorphism $L \longmapsto M^{\prime \prime}$ with $\sigma(L) \neq 0$, thus $\sigma(L) \notin \mathscr{C}$. In this case we conclude that $M \in \rho_{\leq_{\sigma}}(\mathscr{C})$. If $\operatorname{Im}(h) \cap \operatorname{Im}(f) \neq 0$, then there exists $h^{-1}(\operatorname{Im}(h) \cap \operatorname{Im}(f)) \longmapsto M^{\prime}$, a monomorphism with $0 \neq \sigma\left(h^{-1}(\operatorname{Im}(h) \cap \operatorname{Im}(f))\right)$. It follows that $\sigma\left(h^{-1}(\operatorname{Im}(h) \cap \operatorname{Im}(f))\right) \notin \mathscr{C}$, which implies that $\sigma(L) \notin \mathscr{C}$. In this case we conclude that $M \in \rho_{\leq_{\sigma}}(\mathscr{C})$.

Finally, take $\left\{M_{\alpha}\right\}_{\alpha \in X} \subseteq \rho_{\leq_{\sigma}}(\mathscr{C})$. Let us suppose that $f: L \longmapsto \prod_{\alpha \in X} M_{\alpha}$ is a monomorphism with $\sigma(L) \in \mathscr{C}$ and $\sigma(L) \neq 0$. We will show that $\prod_{\alpha \in X} M_{\alpha} \in \rho_{\leq_{\sigma}}(\mathscr{C})$.

We have that $\sigma(L) \stackrel{\sigma(f)}{\longmapsto} \sigma\left(\prod_{\alpha \in X} M_{\alpha}\right) \stackrel{i}{\hookrightarrow} \prod_{\alpha \in X} \sigma\left(M_{\alpha}\right)$ is a monomorphism. Let $\pi_{\beta}$ : $\prod_{\alpha \in X} \sigma\left(M_{\alpha}\right) \rightarrow \sigma\left(M_{\beta}\right)$ denote the canonical projection such that $\pi_{\beta} \circ(i \circ \sigma(f)) \neq 0$. For each $0 \neq R l \in \sigma(L)$ such that $0 \neq \pi_{\beta}((i \circ \sigma(f))(R l))$, as $R l \rightarrow \pi_{\beta}(\sigma(f)(R l))$ is an epimorphism with $R l \in \mathscr{C}$ then $\pi_{\beta}((i \circ \sigma(f))(R l)) \in \mathscr{C}$. This implies that $\pi_{\beta}((i \circ \sigma(f))(R l))=0$. Thus $\pi_{\beta} \circ(i \circ \sigma(f)) \neq 0$ implies $R l=\sigma(R l)=0$, a contradiction. Hence $\sigma(L)=0$. We conclude that $\prod_{\alpha \in X} M_{\alpha} \in \rho_{\leq_{\sigma}}(\mathscr{C})$. Hence $\rho_{\leq_{\sigma}}(\mathscr{C}) \in R-(\sigma$-jtors $)$.

The lattice $R$ - $(\sigma$-Conat $)=\operatorname{Skel}\left(\mathscr{L}_{\{\rightarrow \sigma\}}\right)$, for an exact and costable preradical $\sigma$, is defined in [6]. Where the strong pseudocomplement of $\mathscr{C} \in \mathscr{L}_{\{\rightarrow \sigma}$ is given by

$$
\mathscr{C}^{\left.\perp_{\{\rightarrow \sigma}\right\}}=\{M \in R \text {-Mod } \mid \forall M \rightarrow L, \sigma(L) \in \mathscr{C} \Rightarrow \sigma(L)=0\} \cup \mathbb{F}_{\sigma} .
$$

Proposition 5.8. Let $R$ be a left perfect ring. Let $\sigma$ be an exact and costable preradical. Then for each $\mathscr{C} \in \mathscr{L}_{\{\rightarrow \sigma\}}$, one has that $\mathscr{C}^{\left.\perp_{\{\rightarrow \sigma}\right\}} \in \mathscr{L}_{\{\sigma P\}}$.

Proof. Take $\mathscr{D}=\mathscr{C}^{\perp \rightarrow \sigma \sigma\}}$. Let us see that if $M \in \mathscr{D}$, then $\sigma(M) \in \mathscr{D}$.

Let us suppose that $g: \sigma(M) \rightarrow L$ is an epimorphism with $\sigma(L) \in \mathscr{C}$ and $\sigma(L) \neq 0$. We can complete the diagram: 


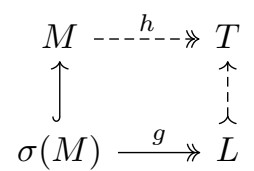

It follows that $\sigma(h): \sigma(M) \rightarrow \sigma(T)$ is an epimorphism, thus $\sigma(T)=L=0$ because $\sigma(L) \in \mathscr{C}$, a contradiction. Hence $\sigma(M) \in \mathscr{D}$.

Let $M \in \mathscr{D}$ and let $g: P(M) \rightarrow M$ be a projective cover of $M$. We are going to show that $\sigma(P(M)) \in \mathscr{D}$.

Notice that $\sigma(P(M))$ is a projective module. Let us suppose that $h: \sigma(P(M)) \rightarrow L$ is an epimorphism with $\sigma(L) \in \mathscr{C}$ and $\sigma(L) \neq 0$. Denote $K=\operatorname{ker}(\sigma(g))$, thus we have the following commutative diagram:

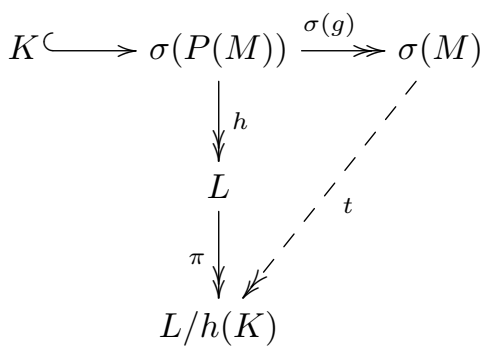

As $\sigma$ is exact we have that $\sigma(L)=L$ and $\sigma(L / h(k))=L / h(K)$, it follows that $\sigma(L) \in \mathscr{C}$ implies that $L / h(K)=\sigma(L / h(K)) \in \mathscr{C}$. As $M \in \mathscr{D}$ and $\sigma(L / h(K)) \in$ $\mathscr{C}$, it follows $L / h(K)=\sigma(L / h(K))=0$. Thus $L=h(K)$. As $\operatorname{ker}(h)+K=$ $\sigma(P(M))$, we have that $\operatorname{ker}(h)=\sigma(P(M))$, which implies that $h=0$ and $L=0$, a contradiction. Hence $\sigma(L) \in \mathscr{C}$ implies that $L=\sigma(L)=0$. We conclude that $\sigma(P(M)) \in \mathscr{D}$.

$R$ is a left Max ring if and only if every conatural class is closed under direct sums (see [2], Theorem 30). Recall that a ring $R$ is left perfect if each left $R$-modules has a projective cover. If $R$ is a left perfect ring, then each conatural class in $R$-Mod is generated by a family of simple $R$-modules (see Corollary 43 of [1]).

Proposition 5.9. Let $R$ be a left perfect ring. Let $\sigma$ be an exact and costable preradical. Then the following statements are equivalent:

(1) $\mathscr{C} \in R$-( $\sigma$-Conat $)$.

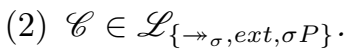

(3) $\mathscr{C} \in \mathscr{L}_{\{\rightarrow \sigma, \oplus, e x t, \sigma P\}}$.

Proof. (1) $\Rightarrow(2)$ We have that if $\mathscr{C} \in R$-( $\sigma$-Conat), then $\mathscr{C} \in \mathscr{L}_{\left\{\rightarrow_{\sigma}, e x t, \sigma P\right\}}$ (see Corollary 4 of [6]).

(2) $\Rightarrow$ (1) Let $\mathscr{C} \in \mathscr{L}_{\left\{\rightarrow_{\sigma}, e x t, \sigma P\right\}}$. We have that $\mathscr{C} \subseteq\left(\mathscr{C}^{\left.\perp_{\{\rightarrow \sigma}\right\}}\right)^{\left.\perp_{\{\rightarrow \sigma}\right\}}$. Take $M \in\left(\mathscr{C}^{\perp_{\{\rightarrow \sigma\}}}\right)^{\left.\perp_{\{\rightarrow \sigma}\right\}}$. We are going to show that $M \in \mathscr{C}$. 
We have that for each $M \in\left(\mathscr{C}^{\perp_{\{\rightarrow \sigma\}}}\right)^{\perp_{\{\rightarrow \sigma\}}}$ and each epimorphism $0 \neq g: M \rightarrow$ $L$ with $\sigma(L) \neq 0$, there exists an epimorphism $0 \neq h: \sigma(L) \rightarrow T$ with $\sigma(T) \in \mathscr{C}$ and $\sigma(T) \neq 0$. Firstly, we show that $\sigma(M) \in \mathscr{C}$.

Assume that $0 \neq g: \sigma(M) \rightarrow L$ is an epimorphism with $\sigma(L) \neq 0$, then there exists an epimorphism $0 \neq h: \sigma(L) \rightarrow T$ with $\sigma(T) \in \mathscr{C}$ and $\sigma(T) \neq 0$. As $\sigma$ is exact, we have that $\sigma(L)=L$, because $L \leq g(\sigma(M)) \leq \sigma(L)$. Besides, $\sigma(T)=T$. Let $f: P(T) \rightarrow T$ be a projective cover, then as $\sigma$ is costable, then $\sigma(P)$ is projective and there exists $f: P(T) \rightarrow \sigma(M)$ such that $f=h g t$ :

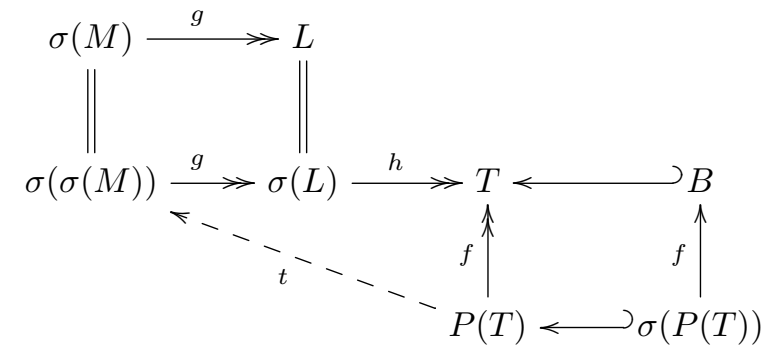

We have that $\sigma(P(T)) \in \mathscr{C}$, then $t(\sigma(P(T)))=\sigma(t(\sigma((P(T))) \in \mathscr{C}$, besides $t(\sigma(P(T))) \leq \sigma(M)$. Let us take $B=f(\sigma(P(T))) \leq T$, it follows that $B=\sigma(B) \in$ $\mathscr{C}$. We have that $B=h g(t(\sigma(P(T))))$, i. e., $B$ is a quotient of $\sigma(M)$, and we get the exact sequence $0 \rightarrow t(\sigma(P(T)) \rightarrow \sigma(M) \rightarrow B \rightarrow 0$, with $t(\sigma(P(T)) \in \mathscr{C}$. This implies that $\sigma(M) \in \mathscr{C}$. Besides, we also have the exact sequence $0 \rightarrow$ $\sigma(M) \rightarrow M \rightarrow M / \sigma(M) \rightarrow 0$, with $\sigma(M), M / \sigma(M) \in \mathscr{C}$ (because $\sigma$ is a radical and $\left.M / \sigma(M) \in \mathbb{F}_{\sigma} \subseteq \mathscr{C}\right)$, which implies that $M \in \mathscr{C}$. We conclude that

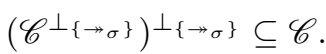

Hence $\mathscr{C}=\left(\mathscr{C}^{\perp_{\{\rightarrow \sigma}}\right)^{\left.\perp_{\{\rightarrow \sigma}\right\}}$. It follows that $\mathscr{C} \in R$-( $\sigma$-Conat $)$. We conclude that $R-(\sigma$-Conat $\left.)=\mathscr{L}_{\{\rightarrow \sigma}, e x t, \sigma P\right\}$.

$(2) \Rightarrow(3)$ Since every left perfect ring is left Max, in this case, every conatural class $\mathscr{C}$ is closed under direct sums, this implies that $\overleftarrow{\sigma}(\mathscr{C})$ is closed under direct sums (see [6], Proposition 4).

As $R$ - $(\sigma$-Conat) $=\{\overleftarrow{\sigma}(\mathscr{C}) \mid \mathscr{C} \in R$-Conat $\}$ (see [6], Proposition 15) we have that all $\sigma$-conatural class is closed under direct sums.

$(3) \Rightarrow(2)$ It is clear.

\section{6. $\sigma$-V-rings and $\sigma$-Max-rings}

We generalize the concept of Max-rings and V-rings.

Definition 6.1. Take $\sigma$ an idempotent preradical. An $R$-module $M$ is $\sigma$-coatomic if each quotient $L$ of $M$ with $\sigma(L) \neq 0$ has a simple quotient $S$ with $\sigma(S)=S$. 
Definition 6.2. Take $\sigma$ an idempotent preradical. A ring $R$ is left $\sigma$-Max if each $R$-module $M$ is $\sigma$-coatomic.

Definition 6.3. Take an idempotent preradical $\sigma$. A ring $R$ is a left $\sigma$-V-ring if each simple $R$-module in $\mathbb{T}_{\sigma}$ is injective.

Theorem 6.4. Let $\sigma$ be a left exact preradical. If $R$ is a left $\sigma$-V-ring, then $R$ is a left $\sigma$-Max-ring.

Proof. Let $f: M \rightarrow N$ an epimorphism where $\sigma(N) \neq 0$. Let us take $0 \neq x \in$ $\sigma(N)$, then $R x$ has a simple quotient $S$ which is $\sigma$-torsion because $\sigma$ is a left exact preradical. Thus, we have a diagram $R x \longleftrightarrow \sigma(N) \longleftrightarrow N$, where ${ }_{R} S$ is an

injective simple module. Then there is a morphism $N \rightarrow S \neq 0$. Hence $M$ is $\sigma$-coatomic and consequently, $R$ is a $\sigma$-Max-ring.

Given a class $\mathscr{C}$ of $R$-modules, we denote $\xi_{\text {conat }}(\mathscr{C})$ the least conatural class containing $\mathscr{C}$ (see [3]), where

$\xi_{\text {conat }}(\mathscr{C})=\{M \in R$-Mod $\mid \forall M \rightarrow N \neq 0, \exists N \rightarrow L \neq 0$ with $L$ quotient of some element of $\mathscr{C}\}$.

Proposition 6.5. Let $\sigma$ be an exact and costable preradical. If $R$ is a left $\sigma$-Maxring, then every $\sigma$-conatural class is closed under direct sums.

Proof. Let $\mathscr{C}$ a $\sigma$-conatural class and let $\left\{M_{i}\right\}_{i \in I}$ be a class in $\mathscr{C}$. We are going to show that for each quotient $L$ of $\bigoplus_{i \in I} M_{i}$ such that $\sigma(L) \neq 0$ there exists a quotient $U$ of $\sigma(L)$ with $0 \neq U \in \mathscr{C}$.

Suppose that $\bigoplus_{i \in I} M_{i} \rightarrow L$ is an epimorphism with $\sigma(L) \neq 0$. As $R$ is $\sigma$-Max, then $L$ has a simple quotient $S$ with $\sigma(S)=S$. As $S$ is also quotient of $\bigoplus_{i \in I} M_{i}$, then $S$ is quotient of one $M_{j}$, for some $j \in I$. As $M_{j} \in \mathscr{C}$, which is a $\sigma$-cohereditary class, and $\sigma(S)=S$, then $S \in \mathscr{C}$. Now $L \rightarrow S$, and $\sigma$-cohereditary imply that $\sigma(L) \rightarrow \sigma(S)=S$, with $0 \neq S \in \mathscr{C}$.

Proposition 6.6. Let $\sigma$ be a left exact preradical. If each $R$-module $M$ is $\sigma$ retractable, then $R$ is a left $\sigma$-Max-ring.

Proof. We have to prove that each module $M$ is $\sigma$-coatomic. Take $M \in R$-Mod and let us suppose that $L$ is a quotient of $M$ such that $\sigma(L) \neq 0$. We will prove that $L$ has a simple quotient $S$ such that $\sigma(S)=S$. 
Let $0 \neq R x \leq \sigma(L)$ be a cyclic module and let us take an epimorphism $g: R x \rightarrow$ $S$, with a simple quotient $S$. As $\sigma$ is left exact, then both of $R x$ and $S$ are of $\sigma$-torsion, i.e., $S=\sigma(S)$. Notice that we have a commutative diagram

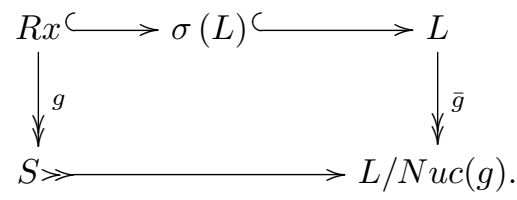

As $L / N u c(g)$ is $\sigma$-retractable and $\sigma(S)=S$ then there exists a nonzero morphism $L / N u c(g) \rightarrow S$, which composed with $\bar{g}$ provides a nonzero $R$-morphism $f: L \rightarrow S$.

We conclude that $M$ is $\sigma$-Max.

Acknowledgement. We thank the referee for the attention devoted to the manuscript and for his detailed, precise and useful comments, which improved greatly the quality of this paper.

\section{References}

[1] A. Alvarado-García, H. Rincón-Mejía and J. Ríos-Montes, On the lattices of natural and conatural classes in R-Mod, Comm. Algebra, 29(2) (2001), 541556.

[2] A. Alvarado-García, H. Rincón-Mejía and J. Ríos-Montes, On some lattices of module classes, J. Algebra Appl., 5(1) (2006), 105-117.

[3] A. Alvarado-García, H. Rincón-Mejía, J. Ríos-Montes and B. Tomé-Arreola, On conatural classes and cotype submodules, Int. Electron. J. Algebra, 11 (2012), 64-81.

[4] A. Alvarado-García, C. Cejudo-Castilla, H. Rincón-Mejía, F. Vilchis-Montalvo and M. Zorrilla-Noriega, On boolean lattices of module classes, Algebra Colloq., 25(2) (2018), 285-294.

[5] L. Bican, T. Kepka and P. Němec, Rings, Modules and Preradicals, Marcel Dekker, Inc., New York, 1982.

[6] E. Cerda-León and H. Rincón-Mejía, Big lattices of module classes induced by preradicals, São Paulo J. Math. Sci., 14 (2020), 185-206.

[7] J. Dauns and Y. Zhou, Classes of Modules, Chapman \& Hall/CRC, Boca Raton, FL, 2006.

[8] S. E. Dickson, A torsion theory for abelian categories, Trans. Amer. Math. Soc., 121 (1966), 223-235.

[9] J. Golan, Torsion Theories, Longman Scientific \& Technical, New York, 1986. 
[10] T. Koşan and M. J. Žemlička, Mod-retractable rings, Comm. Algebra, 42 (2014), 998-1010.

[11] F. Raggi, J. Ríos, H. Rincón-Mejía, R. Fernández-Alonso and C. Signoret, The lattice structure of preradicals, Comm. Algebra, 30(3) (2002), 1533-1544.

[12] H. Rincón-Mejía and M. Zorrilla-Noriega, On some relations between the lattices $R$-nat, $R$-conat and $R$-tors and the rings they characterize, J. Algebra Appl., 12(5) (2013), 1250214 (13 pp).

[13] P. F. Smith, Modules with many homomorphisms, J. Pure Appl. Algebra, 197 (2005), 305-321.

[14] B. Stenström, Rings of Quotients, Springer-Verlag, New York Heidelberg, 1975.

Erwin Cerda-León and Hugo Rincón-Mejía (Corresponding Author)

Departamento de Matemáticas

Facultad de Ciencias

Universidad Nacional Autónoma de México

Circuito exterior, C.U., 04510

Ciudad de México, CDMX, México

e-mails: erwin_nietzsche@hotmail.com (E. Cerda-León)

hurincon@gmail.com (H. Rincón-Mejía) 